Portland State University

PDXScholar

6-5-1985

\title{
The Effect of Subject Expectations of "Hypnosis" Upon the Vividness of Visual Imagery
}

Kayla Mae Nilsson

Portland State University

Follow this and additional works at: https://pdxscholar.library.pdx.edu/open_access_etds

Part of the Psychological Phenomena and Processes Commons, and the Psychology Commons Let us know how access to this document benefits you.

Recommended Citation

Nilsson, Kayla Mae, "The Effect of Subject Expectations of "Hypnosis" Upon the Vividness of Visual Imagery" (1985). Dissertations and Theses. Paper 3566.

https://doi.org/10.15760/etd.5448

This Thesis is brought to you for free and open access. It has been accepted for inclusion in Dissertations and Theses by an authorized administrator of PDXScholar. Please contact us if we can make this document more accessible: pdxscholar@pdx.edu. 
AN ABSTRACT OF THE THESIS OF Kayla Mae Nilsson for the Master of Science in Psychology presented June 5, 1985.

Title: The Effect of Subject Expectations of "Hypnosis" Upon the Vividness of Visual Imagery

APPROVED BY MEMBERS OF THE THESIS COMMITTEE:

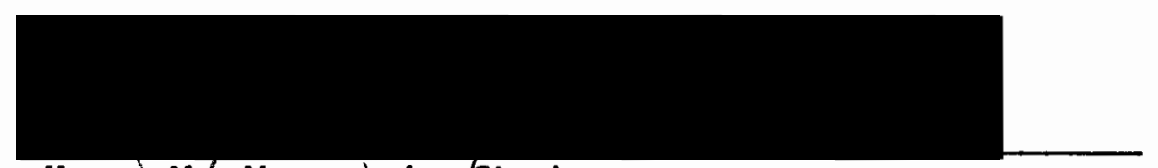

Hugo M. Maynard, /Chairperson

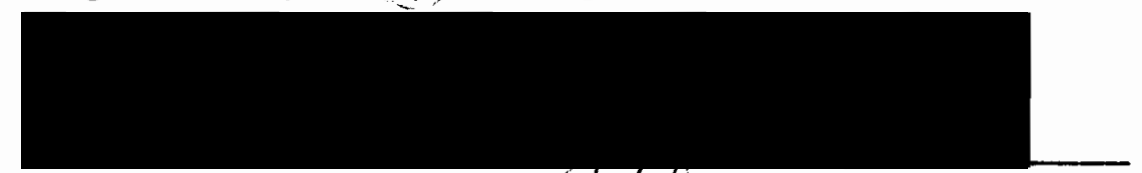

Adriane M. Gaffuri

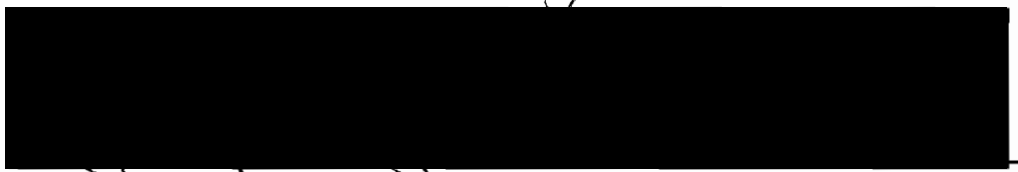

Gary M. Spith

There is no consensus of how hypnosis works. The two major theorists in hypnosis research, the Phenomenologists and the Behaviorists, disagree on this issue. The Phenomenologists consider individual talent and change of the state of consciousness the key to how hypnosis works. The Behaviorists consider the social situation and the roles taken by the experimenter and subject, especially the 
subject's expectations of hypnosis, the mainspring of the hypnotic process. Subject expectations of hypnosis have been found to affect the final results of hypnosis experiments. An experiment was conducted to gain further insight into subject expectations of hypnosis, and how these expectations affect the enhancement of visual imagery (a Behavioral issue) without remaining in the confines of Behavioral theory.

Subject expectations were varied in three experimental groups. All the subjects volunteered for an imagination study and were given a visual imagery test twice. Group 1 expected to be hypnotized just prior to their second imagery test before taking the first (Waking) imagery test. Group 2 did not expect to be hypnotized until just prior to the "hypnosis" exercise and their second imagery test. Group 3 expected to be relaxed just prior to their second imagery test before their first (Waking) imagery test. Finally, the fourth group, used as the control, had no expectations experimentally set; they took two (Waking) imagery tests with a tape on improving study habits placed in between the two tests.

The hypnosis exercise used in the first two groups was actually the same relaxation technique used in the third group, but it was labeled "hypnosis." The relaxation technique was used to test only the expectation of hypnosis, not the effects of hypnosis. 
After all the groups completed the two imagery tests, all the subjects took a hypnotic susceptibility test for group administration (Harvard Group Scale of Hypnotic Susceptibility--Form A). Since previous studies have found a positive correlation between hypnotic ability and the ability to produce vivid visual images, only the subjects who scored 4 or higher were used in the main data analyses.

A total of 63 undergraduates were randomly placed into four experimental groups. Fifty of those individuals were included in the main data analyses.

The present results indicated that there was a significant difference between the first and second imagery test scores for all three experimental groups indicating that vividness of visual imagery was significantly enhanced by the expectation of relaxation in addition to the expectation of "hypnosis," and (in Group 2) imagery was enhanced on the second imagery test even when the subjects did not expect hypnosis during the first test. Descriptive statistics also indicated that the expectation of relaxation increased the enhancement of imagery above the level of the relaxation technique alone, and the expectation of "hypnosis" increased the enhancement of imagery above the level of the enhancement reached by the expectation of relaxation (however, these did not reach statistical significance). No significant evidence was found to indicate that the subjects 
in the experimental groups "held back" on their first (Waking) imagery test to improve on their second imagery test. No significant evidence of the subjects reacting to the word hypnosis was found. A practice effect was found to be an insufficient reason for the improvement in the experimental groups. Finally, a slight indication of hypnotic "slippage" was found. The results also seemed to indicate that a sense of security enhanced hypnotic susceptibility.

These results are discussed in relation to the Behavioral and Phenomenological theories, especially the specific studies this experiment was based on (coe et al., 1980; Lazarus, 1973). Some suggestions for further research are also made. 
THE EFFECT OF SUBJECT EXPECTATIONS OF "HYPNOSIS" UPON THE VIVIDNESS OF VISUAL IMAGERY

by

KAYLA MAE NILSSON

A thesis submitted in partial fulfillment of the requirements for the degree of

MASTER OF SCIENCE

in

PSYCHOLOGY

Portland State University

1985 
TO THE OFFICE OF GRADUATE STUDIES AND RESEARCH:

The members of the Committee approve the thesis of Kayla Mae Nilsson presented June 5, 1985.
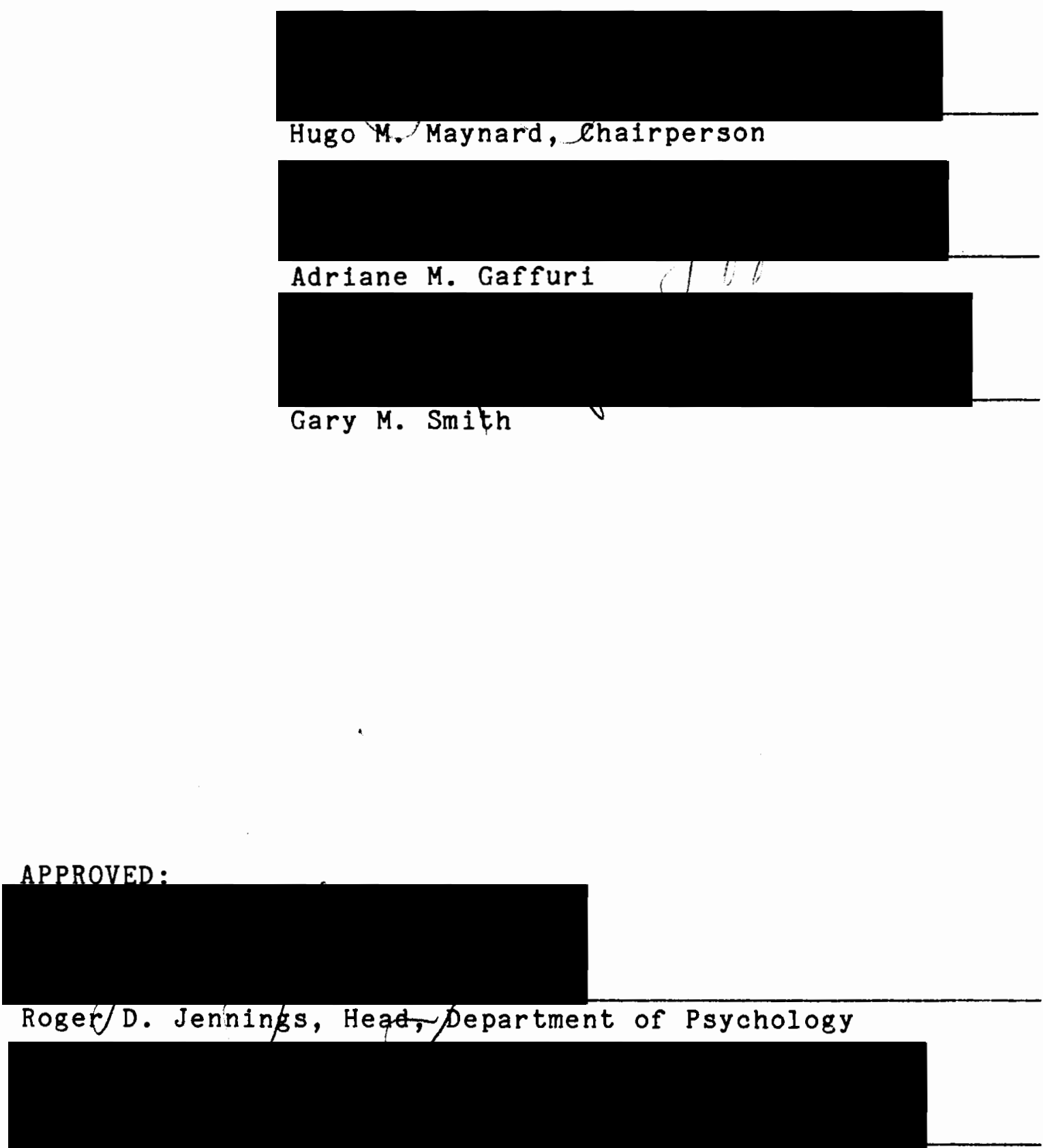

Jim F.CHeath, Dean of Graduate Studies and Research 
TABLE OF CONTENTS

PAGE

LIST OF TABLES • . . . . . . . . . . . . . . . . v

LIST OF FIGURES . . . . . . . . . . . . . . . . vi vi

CHAPTER

I INTRODUCTION . . . . . . . . . . . . 1

Subjects' Expectations in Hypnosis

Experiments............. . 2

Enhancement of Visual Imagery in

Hypnosis Experiments . . . . . . . 12

Goals of the Thesis and Design

of the Study . . . . . . . . . . 17

Predictions Indicated. . . . . . . 20

II METHODS . . . . . . . . . . . . . 25

Subjects . . . . . . . . . . 25

Measures............. 26

Procedure. . . . . . . . . . 30

III RESULTS AND DISCUSSION . . . . . . . . . 33

Outcome of the Predictions . . . . . 36

The Sample. . . . . . . . . . . 46

The Relaxation Technique Used . . . . 51

Lazarus (1973) . . . . . . . . 52

Coe, St. Jean, and Burger (1980) .... 55

Conclusions ............ 58

Summary of Findings . . . . . . . 62 
Page

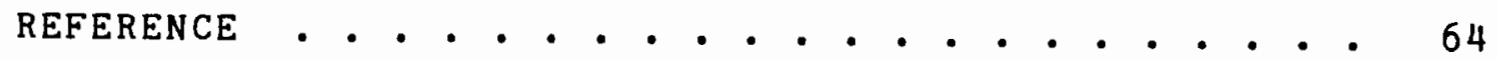
APPENDICES

A Diagram of the Design . . . . . . . . . 71

B Health Status Questionnaire . . . . . . . 72

C Consent Forms Used . . . . . . . . . . 73

D Measures Used . . . . . . . . . . . 78

E Procedures Used . . . . . . . . . . . . 94

F Raw Data and Subject Information . . . . . 114

G Figure 2. Mean VCI Scores for Low

Susceptible Subjects . . . . . . . 118 


\section{LIST OF TABLES}

TABLE

PAGE

I Summary of Study by Stam and Spanos . . . . . 6

II Percentages of Subjects in Each Group With

Tendencies to Have Vivid Visual Images . . 48 


\section{LIST OF FIGURES}

FIGURE

PAGE

1. Mean VCI Scores for All Groups;

HGSHS-A Scores 4 and Above....... . 34

2. Mean VCI Scores for Low Susceptible

Subjects HGSHS-A Scores 0-3 . . . . . 118 


\section{CHAPTER I}

\section{INTRODUCTION}

Scientists have studied hypnosis for hundreds of years. Faith healers and other spiritualists, past and present, have used and are using hypnosis; yet, there is no consensus on how hypnosis works, if it is a distinct state, or exactly what it does.

This lack of understanding may be due, partly, to the way the study of hypnosis has been approached. There are two major theoretical orientations in hypnosis research according to Fromm and Shor (1972): Phenomenological and Behavioral. These different orientations not only ask different questions and expect different answers; they operate under different philosophical systems which guide them in different directions.

Phenomenologists studying hypnosis believe the ultimate issue to be explained is the subject's inner experience. They study unconscious mental functioning, the depth of the hypnotic trance, and how and why hypnosis changes the state of consciousness; they tend to study one or two individuals with proven hypnotic ability over a long period of time. They propose that hypnotic susceptibility and talent of the subjects are more important than the expertise 
of the experimenter (i.e., the subject has the talent to hypnotize him/herself, and the "hypnotizer" is more of a teacher than a master).

On the other hand, Behaviorists studying hypnosis believe the ultimate issue (the only issue) is the observable behavior of the subjects. Behaviorists tend to study a great number of inexperienced subjects for a short period of time and believe the effects of hypnosis are due to the roles the subject and experimenter take: the subject is "hypnotized" because he/she expects to be hypnotized and is persuaded by the setting and the experimenter's prestige (Fromm \& Shor, 1972).

This thesis does not intend to prove either orientation more correct. It does intend to examine the effect that subject expectations have on hypnosis (a Behavioral issue) without remaining solely in the confines of the Behavioral orientation.

\section{Subjects' Expectations in Hypnosis Experiments}

Orne (1959) demonstrated that the subjects' preconceptions about hypnosis affected their subsequent behavior during hypnosis. In a psychology class subjects were given the impression that catalepsy of the dominant hand was an indication of trance behavior. The subjects were then hypnotized, and 5 out of 9 volunteers showed catalepsy in either hand. In a later article orne (1967) stated that if 
all of the subjects' expectations of hypnosis are controlled for, there is no physical evidence that hypnosis is present (i.e., all hypnotic phenomena are caused by expectation). Brown and Krasner (1969) also found that subjects' expectations affected subsequent hypnotic experience. In their study they let two groups of subjects view one of two video tapes of a model being hypnotized prior to the subjects' experience with hypnosis. The first group viewed the videotape in which the hypnotic induction went from easily accomplished tasks (e.g., hand lowering) to very difficult tasks (e.g., auditory hallucinations) and then were hypnotized. The second group viewed the videotape in which the difficulty in hypnotic tasks went from very difficult to very easy and then were hypnotized.

The experimenters assumed that the subjects in the first group would expect hypnosis to be easy, and the subjects in the second group would expect hypnosis to be hard. Although the a posteriori $2 \times 2$ analysis of variance they used did not reveal significant effects, because of the pattern of results they

- . cautiously concluded that the results of the present study lend some support to the hypothesis that responsivity to suggestions given in the context of a hypnosis experiment can be influenced by manipulating expectations and structuring the hypnosis session so that these expectations are either confirmed or disconfirmed (Brown \& Krasner, 1969 ; p. 186). 
Another study (Stam \& Spanos, 1980) found expectations affected the outcome of their hypnosis experiment. They tested the subjects' hypnotic susceptibility; then, randomly assigned 40 subjects who were highly susceptible into one of four groups. All the subjects had one of their hands immersed in ice water three times for 60 seconds each and were asked to rate the magnitude of their pain. The first immersion was used as a baseline assessment and was the same for all four groups.

In Group 1 the subjects were told they would receive two more immersions, and that they would be hypnotized during the third immersion. Then each subject's alternate hand was immersed in the ice water with only the suggestion that they would not feel any pain. Finally, they were hypnotized and given the same suggestion used for the second immersion; then, they had their first hand immersed in the ice water again.

In Group 2 the subjects were told they would not be hypnotized again, and that the two other immersions would be preceded by a waking analgesic suggestion (i.e., the same suggestion used in Group 1), and then their alternate hand was immersed in the ice water followed by their first hand for the third immersion.

In Group 3 the subjects were told that hypnosis would probably be less effective in reducing pain than a waking analgesic suggestion. Then they were hypnotized again and 
had their alternate hand immersed. Finally, they were given the analgesic suggestion by itself and had their first hand immersed again.

In Group 4 the second and third immersions (using alternating hands) followed the same procedure as the baseline immersion.

The results indicated that Group 1 had a smaller decrease in pain for the waking analgesic suggestion than for the hypnotic analgesic suggestion. In Group 2 the subjects' pain estimations during the second and third immersions with the waking suggestion were comparable to the pain estimations of the subjects in Group 1 during the hypnotic suggestion. This indicates the subjects in Group 1 may have "felt" more pain during the second (Waking) immersion because they were expecting to feel less during the third immersion under hypnosis. In Group 3 the subjects (who were told that hypnosis was not effective and were given a hypnotic analgesic and then a waking analgesic suggestion) reported more pain during both the hypnotic immersion and the waking immersion than Group 1 did during either the waking or the hypnotic immersion, or than Group 2 did during both waking immersions. There were no differences in the pain estimations for the three immersions in Group 4. This experiment is summarized in Table $I$. 


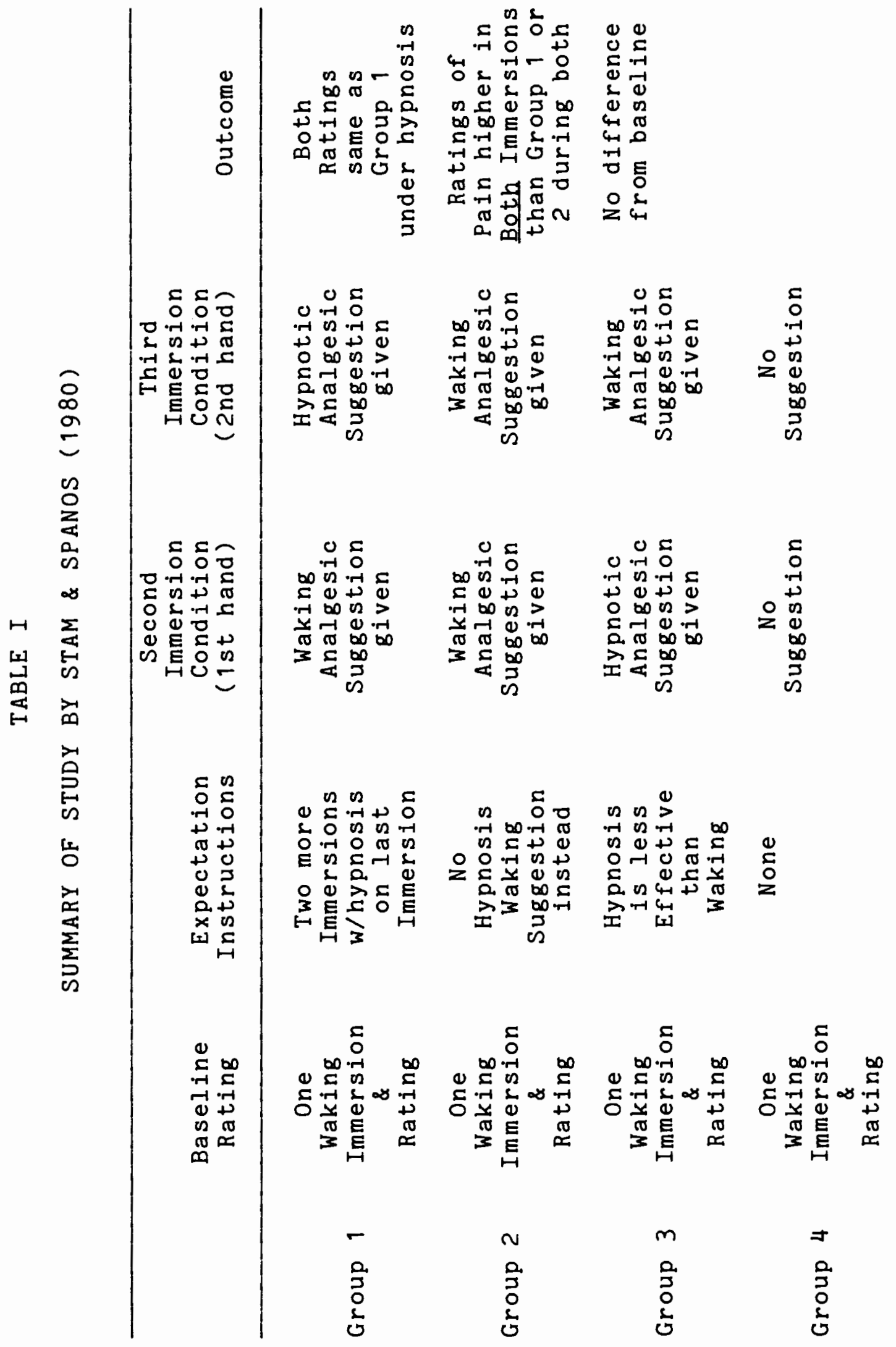


In a clinical setting Lazarus (1973) administered a relaxation exercise to 20 clients who requested hypnosis. However, he told half of his clients that the procedure was a relaxation technique, and he told the other half that the same procedure was a hypnotic induction. He found that the clients who received the "hypnosis" they requested showed greater improvement in therapy and had more vivid visual imagery than did the clients who received the correctly labeled relaxation technique. As a control, he gave 9 clients who did not request hypnosis both the relaxation and "hypnosis" conditions and found these clients reported no difference between the two procedures.

Because this study was conducted within a clienttherapist relationship, there were some strategic flaws within its design: (1) All the "experimental" subjects requested hypnosis. Since the client's expectations were not uniformly set and controlled, these expectations cannot be considered equivalent. (2) such phenomena as transference and other parts of the client-therapist relationship could have had a great affect on the outcome (i.e., When a therapist tells a client no that he/she will not receive what he/she requested, it is much different than when an experimenter, with no previous relationship with that person, tells him/her no). (3) The "control" group received both conditions without asking for either. This is inadequate because when both procedures are given to the 
same person, it is hard to differentiate what is caused by the particular condition, and what is caused by a practice effect. In addition, when people are in therapy with problems of their own, it would seem that they would not put much effort into something they did not ask to do.

Although the Lazarus study was not well-designed, it did focus attention, as did the studies preceding it, on a very important and somewhat neglected issue in hypnosis research: the effect of subjects' expectations of hypnosis upon hypnosis.

Coe, St. Jean, and Burger (1980) also addressed the question of how subject's expectations affect the outcome of hypnosis experiments. Unlike the Lazarus study, however, they did it within a better controlled and designed experiment.

Their 1980 study consisted of two experiments. In the first, all the subjects volunteered for a hypnosis experiment. During the first session of this experiment the Stanford Hypnotic Susceptibility Scale Form A (SHSS-A) (Weitzenhoffer \& Hilgard, 1959) was administered. Then the subjects were randomly assigned to two groups for the second session. In both groups the subjects took the same visual imagery test, the Vividness and Control of Imagery (VCI), adapted from items used in Marks (1973), two times: once with waking imagination instructions, and once under 
hypnosis with hypnotic imagination instructions ( 6 items out of the 12 items from the SHSS-form C, Weitzenhoffer \& Hilgard, 1962, were used for the hypnotic induction). In the first group the subjects were told they would take an imagery test first and be hypnotized later. The VCI with waking imagination instructions was given first and then the hypnotic VCI. In the second group the order of presentation was reversed. The subjects in the second group, apparently, were not told about the second (Waking) imagination test until after they had taken the first, hypnotic VCI. A significant difference between the waking and the "hypnotic" VCI scores was found, but only when the waking VCI was given before the hypnotic VCI (i.e., the first group). There was no significant difference in scores for the second group in which the waking VCI was given after the hypnotic VCI. Coe et al. suggest that the difference between the first and second groups may have been due to the enhancement of ability during hypnosis [in the second group this hypnotic enhancement may have been carried over to the second "waking" VCI], or a practice effect of taking the same imagery measure twice.

After the end of the experiment, the subjects were divided into three groups according to their hypnotic susceptibility scores (the 6 items from the SHSS-A were transformed to a 12 point scale): A score of $0-4$ was low; 5-8 was medium, and 9-12 was high. Each of the two 
preceding groups had approximately an equal number of subjects from each of the three susceptibility levels. Their results indicated that there was also a significant difference between the VCI scores of the subjects in the first group (Waking-First) who scored high, medium, or low. The subjects with high and medium scores did significantly better on both VCI tests than did the subjects within the low susceptibility range. In the second group (HypnosisFirst) the same trend occurred but did not reach significance.

In the second experiment in coe et al.'s study a different sample of subjects volunteered for an imagination experiment, and then were treated in one of two ways. In the first group (Waking) the subjects were, again, told the experimenter was interested in imagination. The subjects then took the VCI with waking imagination instructions. Then the experimenter told the subjects he/she was also interested in hypnosis and the subjects took the 8 items of the SHSS-C which were used in the first experiment. The same 6 items used in the first experiment were also transformed to a 12 point scale and used to obtain an indication of the subjects' hypnotic susceptibility level. In the second group (Hypnosis) the subjects were told the experimenter was interested in hypnosis as well as imagination. The subjects then took the same 8 items of the SHSS-C used in the first group, the hypnotic instructions, and the 
VCI. As in the first group, 6 of the 8 items from the SHSSC were used to assess hypnotic susceptibility.

There was no significant difference between the VCI scores of the Waking and Hypnotic groups. However, there was a significant difference in scores between the three susceptibility levels (the same outcome as the first experiment): the high and medium subjects performed better on both VCI tests than did the low subjects.

Although the Coe et al. study directed some attention to the effect of subject expectations, it was not conclusive, even though the first experiment provided data concerning subject expectations that could have been analyzed. The data from the first experiment were analyzed by two separate ANOVA's which tested the effects of hypnosis and the waking imagination exercise on the VCI scores, and the effect of the level of susceptibility on the VCI scores. If they had analyzed the same data with one, three-way ANOVA, adding the factor of the order of presentation (i.e., Waking-First, or Hypnosis-First), they would have been able to make a more statistically sound conclusion about the effect of subject expectations.

The present thesis combined the essential components of the two experiments in the coe et al. study (The WakingFirst group found in their first experiment and having all the subjects volunteer for an imagination experiment found 
in their second) with the essential component of the Lazarus study (1973) (comparing the effect of relaxation correctly labeled to the effect of relaxation labeled "hypnosis"), previously mentioned, and focused the analysis on the effect of subject expectations within a controlled experiment.

One of the major benefits of hypnosis is its potential capacity to enhance imaginative ability. If subject expectations are the primary cause for the changes which occur during hypnosis, then therapists using hypnosis to enhance visual imagery can focus on setting expectations rather than focusing on the hypnotic induction itself, and more research can also be focused on how and why expectations affect hypnosis.

\section{The Enhancement of Visual Imagery in Hypnosis Experiments}

The Phenomenologists see the enhancement of imagery during hypnosis as evidence of an alteration in the individual's state of consciousness. This orientation's description of an altered state of consciousness is typified by Shor's concept (1959 cited in Sheehan, 1972), the Generalized Reality-Orientation, and its disintegration during hypnosis:

The concept of Generalized Reality-Orientation refers to the background of awareness, the frame of reference that denotes the context within which one interprets all ongoing conscious experiences. Shor argues that under hypnosis this orientation to reality fades into the background so that ongoing experiences become relatively isolated from their 
usual frame of reference. The structure of the Generalized Reality-Orientation permits what in Freudian terminology is called secondary process thinking. Viewed in this way the ability temporarily to give up reality orientation corresponds to a regression to primary-process functioning.

With the loss of the Generalized RealityOrientation, the distinction between reality and imagination fades and primary process modes of thought such as imagery and fantasy are allowed to flow more easily into awareness [italics added] (Sheehan, 1972; p. 298).

The Behaviorists do not believe that enhancement of imagery is evidence of an altered state of consciousness, but some Behaviorally-oriented therapists have found that hypnosis would be a useful therapeutic tool (Coe et al., 1980). The ability to enhance visual imagination is useful in Behavior therapy because it allows the hierarchy schemes used in systematic desensitization to become more real to the clients (Dengrove, 1973; and Wolpe, 1969). Vivid visual images also improve the effectiveness of covert conditioning (Cautela, 1975).

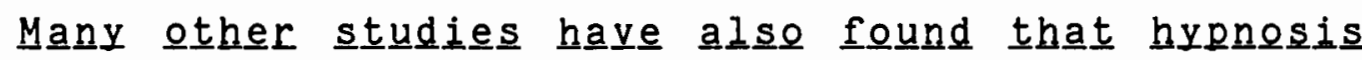
enhances visual imagery. Kroger and Felzer (1976) measured the vividness of imagery of the clients they saw in therapy, and because the clients improved, and hypnosis was used, they came to the conclusion that hypnosis enhanced visual imagery. Rossi, Sturrock, and Solomon (1963) measured imagery by using 31 multiple-choice questions pertaining to the vividness and clarity of 11 standard images (e.g., name in neon lights, familiar car, etc.). They found that 
imagery was enhanced in the hypnotic condition. In the Sanders study (1969) subjects were randomly assigned to one of two groups (hypnosis or waking) and both these groups were asked to imagine five figures described to them and then to draw them. Their results also supported the hypothesis that hypnosis enhances visual imagery. Finally, Stross and Shervin (1962) showed their subjects a rebus stimulus, a picture of a tie and a knee, while hypnotized or while awake and made transcripts of the subjects' verbalizations, looking for any mention of "tiny" (i.e., "tie-knee), "small," or "little" images; thereby, showing an unconscious recognition of the rebus meaning. They found that the subjects who saw the rebus stimulus while hypnotized had a significantly greater number of "small," "little," or "tiny" images.

other studies have found that individuals who are more

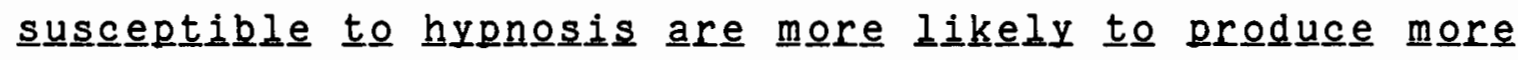
vivid visual images. Two experiments (Diamond \& Taft, 1975; Wagman \& Stewart, 1974) which used the Betts Questionnaire upon Mental Imagery ( $Q M I$ ) found that the ability to see images vividly correlated positively with hypnotic susceptibility. Shor, Orne, and O'Connell (1966); Spanos, Churchill, and McPeak (1976); Spanos, Valois, Ham, and Ham (1973); and Sutcliffe, Perry, and Sheehan (1970) also found a positive correlation between hypnotic susceptibility and vividness of imagery using different, shortened variations 
(15-35 items) of the Betts QMI (150 items). Finally, Palmer and Field (1968) also found a positive correlation between hypnotic susceptibility and vividness of imagery even during a waking state. After the experimenters tested the subjects' susceptibility level, the subjects were asked to describe all images seen during a waking state, and the more susceptible subjects saw more vivid images.

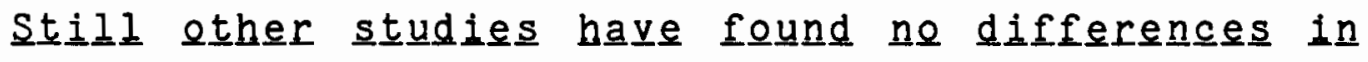

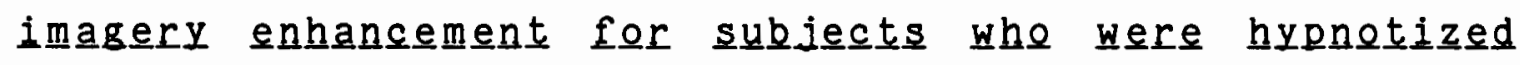
compared to those who received task-motivating instructions. Ham and Spanos (1974) measured imagery by asking their subjects to rate the vividness of one image on a scale of 1 to 5 and found that the hypnosis group and the taskmotivation group did not differ in their performances. The task-motivation instruction encouraged the subjects to imagine the suggested effects vividly and informed them that the suggestions were easily accomplished, and other subjects were easily able to pass them. Starker (1974) primed his subjects by letting them listen to a tape-recording about how to form mental images and then gave them 15 stimulus words and asked them to rate the vividness of each on a scale from 1 to 5 . He found that the hypnotic procedure used did not significantly increase the vividness of imagery beyond that produced in the motivational or control groups. In the Wadden and Flaxman study (1981) the experimenter- 
therapist (he also counseled the subject about problems they were having losing weight) asked the subjects to describe the clarity of their visual imagery in the scenes used to promote weight loss and found no significant difference between the hypnosis, covert modeling, or relaxation groups. These studies which compared hypnosis to taskmotivational instructions, however, did not use standardized measures of imagery nor did they stratify their samples according to hypnotic susceptibility (Coe et al., 1980) or even measure these susceptibility levels. In addition, the Wadden and Flaxman study (1981) was also complicated by the client-therapist relationship discussed earlier.

However, Coe et al. (1980), the study which tested subject expectations, stratified the hypnotic susceptibility levels of their subjects and measured how hypnosis affected the vividness of visual imagery compared to how the waking imagination instructions affected the vividness of visual imagery in their two experiments.

In their second, the authors found no difference in imagery enhancement for the subjects who were randomly assigned to the Waking-only condition as opposed to the Hypnosis-Only condition. However, in the first experiment of the same study in which both the waking VCI and the hypnotic VCI were given to each subject, a significant difference was found for the Waking-First group but not for the Hypnosis-First group. The subjects in the Waking-First 
group produced significantly higher scores on their hypnotic VCI tests than they did for their waking VCI tests. In other words, subjects who were expecting to be hypnotized but were given the waking VCI first were more affected than the subjects who were hypnotized first and then took their waking VCI.

\section{Goal of the Thesis and Design of the Study}

This thesis was formulated to examine why the WakingFirst Condition in Coe et al. (1980) was the only group in their first experiment that had significantly different VCI scores for the waking and hypnotic administrations and to particularly focus on what effect subject expectations had on this first experiment of the coe et al. study.

To be certain that the scores on the VCI are due to expectation of hypnosis, not "real" hypnosis, no hypnotic induction will be used. Instead, the relaxation technique used in the Lazarus study (1973) will be used for all three experimental groups. This relaxation technique will be either correctly labeled relaxation or labeled "hypnosis." The only difference between these two exercises is the insertion of two sentences from the SHSS-A between the first and second paragraph (See Appendix D).

Specifically, different expectations are set for each of the four groups (please see the diagram of the design; 
Appendix A). All the subjects will volunteer for an imagination experiment (See Appendix E for recruitment procedure) and, therefore, will not have any expectations of hypnosis before coming to the experiment.

In Group 1 the subjects will be informed about the "hypnosis" at the beginning of the session and will expect hypnosis while they are taking the waking VCI. Then they will be "hypnotized" and take their "hypnotic" VCI (this group is the equivalent of the Waking-First group in coe et al., 1980, except that the present experiment will not use "real" hypnosis). In Group 2 the subjects will not expect to be hypnotized until after they have taken the waking VCI. They will be informed about the "hypnosis" just prior to being "hypnotized"; therefore, their expectation of hypnosis will not affect their first VCI scores, as it will in Group 1 , because the subjects will not know about the "hypnosis" during their waking VCI. In Group 3 the subjects will be informed about the relaxation at the beginning of the session and will expect relaxation while they are taking their waking VCI. Then they will be relaxed and take their relaxed VCI; therefore, their waking VCI will be affected by the expectation of relaxation which will occur later in the session. In Group 4, the control group, the subjects will take two waking VCI tests. In between the two VCI administrations the subjects will listen to a tape on improving their study habits, the SQ4R (See Appendix D). This will be 
used in place of the relaxation of "hypnosis" used in the other three groups. No expectations will be set in this group; therefore, only a practice effect could cause any significant difference in scores between the first and second VCI administrations.

After the two VCI tests are given in the four groups, all the subjects will take the Harvard Group Scale of Hypnotic Susceptibility Form A (HGSHS-A) (Shor \& Orne, 1962) to assess their level of hypnotic susceptibility. Because Coe et al. (1980) found that their subjects who had a medium or high degree of hypnotic susceptibility (5-12 on the SHSSC) produced significantly higher scores on both VCI administrations than the subjects who had a low degree of hypnotic susceptibility ( $0-4$ on the SHSS-C), this study will use only the data from subjects who receive at least a $4^{1}$ on the HGSHS-A in the main data analyses. This will give some assurance that the differences in the VCI scores will be less affected by the level of hypnotic susceptibility of the

${ }^{1}$ Although in Coe et al. (1980) the 6 items from the SHSS-C used to assess hypnotic susceptibility were transformed to a "12" point scale, and the HGSHS-A (used in the present experiment) used a 13 point scale (so they can be considered comparable), this experiment used different susceptibility ranges for $10 \mathrm{w}$ and medium (i.e., $0-3$ is low and 4-8 is medium) because only some evidence of ability to produce vivid visual images was needed (this ability did not need to be great), and because the number of subjects was limited. 
subjects, and that the subjects used in the main data analyses will be able to produce reasonably vivid visual images.

\section{Predictions Indicated}

\section{Prediction 1}

If subject expectation is a key factor in this experiment, then the waking VCI scores in Group 1 (expect-hypnosis during waking VCI) will be lower than the waking VCI scores in Groups 2 and 4 (i.e., in both of these groups the subjects have no expectations of hypnosis while taking their first, waking VCI) because the subjects' expectations in Group 1 will cause them to "hold back" on the waking VCI test in order to get a higher score on the subsequent "hypnotic" VCI test. This was found and was predicted by Scharf and Zamansky (1963) and Zamansky, Scharf, and Brightbill (1964) but did not occur in Coe et al. (1980).

\section{Prediction 2}

The same "holding back" effect may also occur in Group 3 (in which the subjects' will expect relaxation during the waking VCI) but not to the same extent as it will in Group 1 (expect-hypnosis) because merely the mention of the word hypnosis should cause a great deal of expectation (Orne, 1967). Orne's findings predict that the label "hypnosis" will cause a greater amount of expectations for the subjects than the label "relaxation." Therefore, the waking VCI 
scores in Group 3 (expect-relaxation) will be higher than the waking VCI scores in Group 1 but lower than the waking VCI scores in Groups 2 and 4 because the subjects in Group 3 will "hold back" on their first VCI because of expectation, and because the subjects in Groups 2 and 4 will have no expectations set during their first VCI.

\section{Prediction 3}

In the same vein, if the expectation of hypnosis is a key factor in this experiment, not just expectation in general, then there will be a greater difference between the waking VCI scores and the "hypnotic" VCI scores in Group 1 (expect-hypnosis) than between the waking VCI scores and the relaxation VCI scores in Group 3 (expect-relaxation). The preceding is predicted because the "hypnotic induction" used in Group 1 is also the relaxation technique used in Group 3; Hamberger and Lohr (1980) found that visual imagery was not increased during relaxation. In fact Rehm, Mattei, Potts, and Skolnick (cited in Hamberger \& Lohr, 1980) found that relaxation training actually decreased the frequency of vivid images of their subjects. Hence, if imagery is enhanced on the second, "hypnotic" VCI in Group 1 (indicated by a higher score), then it must be caused by the subjects' expectation of hypnosis, since relaxation does not increase vividness of imagery when labeled correctly. 
Prediction 4

If the mere mention of the word hypnosis heightens people's expectations immediately, as some Behaviorallyoriented researchers would predict (Sarbin \& Coe, 1972), then the first VCI scores in Group 1 (expect-hypnosis during the first VCI) will be higher than the first VCI scores in Groups 2, 3, and 4 because the subjects in Group 1 will know about the "hypnosis" before they take the first VCI, and the subjects in Groups 2, 3, and 4 will not. The second VCI scores in Group 2 will be relatively equal to the second VCI scores in Group 1 because by the time the second VCI is given in Group 2, these subjects will expect hypnosis and catch up with the subjects in Group 1. Both groups' ( 1 and 2) scores for the second, "hypnotic" VCI will be higher than the second VCI scores for Groups 3 and 4 because these groups will not expect hypnosis during any part of the imagery testing.

\section{Prediction 5}

If subjects' expectations have no effect on the enhancement of vividness of visual imagery, then the difference between the first VCI and the second VCI scores in Groups 1,2 , and 3 will not differ significantly from the difference between the first VCI and the second VCI scores in Group 4. In other words, the only difference between the two VCI administrations for each of the four groups will be 
due to a practice effect. (Please see Prediction 6 for further discussion.)

\section{Prediction 6}

Tart (cited in Fromm \& Shor, 1972) stated that a change in the state of consciousness, such as hypnosis, can appear without any special procedures, such as a hypnotic induction. Andreychuk and Skriver (1975) and Knox and Shum (1977) found that highly susceptible subjects may become hypnotized without any formal induction (i.e., highly susceptible subjects can "slip" into a hypnotic state).

If highly susceptible subjects can become hypnotized without any special procedures (which is termed "hypnotic slippage"), as these Phenomenological researchers predict and found, then two factors must be examined.

First, looking at the highly susceptible subjects only (i.e., individuals who scored 9 or higher on the HGSH-A), the second VCI scores in Group 4 will be significantly higher than the first VCI scores in the same group if anyone "slipped" into a hypnotic state.

Second, the second VCI scores of the medium susceptible subjects ( $4-8$ on the HGSHS-A) in Group 4 will not be significantly different from their first VCI scores. This second factor needs to be examined to test whether a practice effect will raise scores in Group 4 for the medium susceptible group. If it does not, one can assume that the 
VCI scores in the highly susceptible group will not be raised by anything other than "hypnotic slippage."

In addition, the SQ4R tape will be used in Group 4 to keep the subjects more alert (i.e., in a normal state of consciousness) either because of their interest in the SQ4R passage or their annoyance with it. In this way only highly susceptible subjects will be apt to "slide" into hypnosis.

The VCI scores in Group 4 are the only scores which can be checked for hypnotic "slippage" in this design because it is the only group in which no expectations will be set for either of the two VCI administrations. "Slippage" into hypnosis is also possible in the other three groups. However, because the other three groups will involve expectation of either "hypnosis" or relaxation, it would be difficult to verify that changes in these VCI scores were caused by hypnotic "slippage" instead of expectation. 
CHAPTER II

ME THODS

\section{Subjects}

A total of 63 subjects ( 35 women and 28 men) were selected from volunteers enrolled in psychology classes at Portland State University. Extra-credit was offered in most of the classes in exchange for their participation. No other compensation was offered (see Appendix $E$ for the recruitment procedure).

At the time of recruitment, volunteers were given the Health Status Questionnaire (see Appendix B). This was used as a screening device to detect anyone who might have been disturbed by any aspect of the experiment. If the volunteers passed this screening procedure, they were called for an appointment and randomly assigned to one of the four groups. Of the 129 volunteers, 39 people were not included in the sample because they did not pass the screening device; 27 people passed this device but either could not schedule an appointment or did not keep their scheduled appointment.

The age range of the 63 subjects who passed the screening device and kept their appointments was 19 to 49 years with a median age of 25 years and a mode of 20 years 
of age. From these 63 subjects 50 subjects ( 27 women and 23 men) were included in the main data analyses because they scored above 3 on the HGSHS-A. Eleven subjects ( 7 women and 4 men) scored 3 or below on the HGSHS-A and were only included in the data analyses where it is specifically stated. Two subjects ( 1 woman and $1 \mathrm{man}$ ) did not answer all of the questions on the two VCI tests given and could not be included in any of the data analyses (nor were they included in Appendix F). All of the subjects who were asked to be hypnotized agreed.

\section{Measures}

All the subjects were given (1) the vividness section of the Vividness and Control of Imagery scale (VCI) modified for group administration (see Appendix D), (2) the waking imagination instructions used in coe et al. (1980), and (3) the Harvard Group Scale for Hypnotic Susceptibility Form A (HGSHS-A) (Shor \& Orne, 1962).

Groups 1, 2, and 3 were given the hypnotic imagination instructions also found in Coe et al. (1980). However, in Group 3 all references to hypnosis were changed to refer to relaxation (e.g., "relaxation" imagination instructions). In addition, Groups 1, 2, and 3 were given the relaxation technique found in Lazarus (1971), but, as stated before, this exercise was labeled either "relaxation" (for Group 3) or "hypnosis" (for Groups 1 and 2) and is exactly the same 
except for two sentences in which only two words are different (see Appendix $D$ ). The two sentences were taken from the SHSS-A (Weitzenhoffer \& Hilgard, 1959).

The first section of the VCI was selected to test the vividness of visual imagery in order to make it comparable to coe et al. (1980). This first section consisted of a total of 15 questions which the subjects answered after they were given a scene to imagine (there were four scenes). Each question was answered on a rating scale of one to five: five meant the image was very vivid, and one meant the image was not very vivid at all. The present experiment used the first scene (and its 4 questions) as a rehearsal because some of the subjects were still confused after the taped instructions; therefore, 11 items were used in the data analyses whereas, Coe et al. (1980) used all 15 items in their data analyses.

The Harvard Group Scale of Hypnotic Susceptibility Form A (HGSHS-A; Shor \& Orne, 1962), a self-report hypnotic susceptibility scale is an adaption of the Stanford Susceptibility Scale (SHSS) by Weitzenhoffer and Hilgard (1959) used in Coe et al. (1980). Bentler and Hilgard (1963) and Shore and Orne (1963) found that, even though scores on such self-reports (as the HGSHS-A) tended to be a little higher than objective reports (the SHSS), the results were very 
comparable (correlations were $r=.81$ and $r=.70$, respectively).

O'Connell (1964) found that susceptibility scores derived from self-ratings were in excellent agreement with objective ratings $(r=.90)$; and that poor hypnotic subjects tended to under-evaluate their performances, and good hypnotic subjects tended to over-evaluate their performances. Therefore, the HGSHS-A should give a good indication of hypnotizability. Since an ability to be hypnotized is positively correlated with the ability to produce vivid visual images, the HGSHS-A should also be a good indicator of the ability to produce vivid visual images.

To keep the control group, Group 4, equivalent to the other three groups, the subjects in this group listened to a passage on the SQ4R (i.e., Study--Question--Read--Recite-Write--and Review) by Siebert and Walter (1983) (see Appendix D). This passage was used in between the two waking VCI administrations and was approximately the same length ( 8 minutes) as the relaxation technique used in the other three groups. These instructions on improving study habits were designed to engage the attention of the subjects (who were all students) with a "non-hypnotic" target for their concentration.

The HGSHS-A, the waking and hypnotic instructions, "relaxation" instructions, "hypnosis induction" and relaxation technique, the two VCI tests, and the SQ4R were offered 
on audio-tape in order to present the material the same way each time. To further insure that the sessions were uniform, these procedures (except for the HGSHS-A) were placed on reel-to-reel tape, first, and transferred to cassette tape in the correct order for each group; therefore only one version of each procedure was given to all four groups. The HGSHS-A was placed directly on one cassette tape and played for each of the groups since it was presented last in each group. Estrabrook (1930) and Hull (1933) found that taped hypnotic inductions worked as well as live inductions.

Each of the four groups received a series of consent forms relevant to their experimental condition. Group 1 was given a hypnosis consent form at the beginning of the experiment. Group 2 was given a general consent form before the first VCI and a hypnosis consent form after the first VCI. Group 3 was given a relaxation consent form at the beginning of the experiment and a hypnosis consent form after the second VCI. Group 4 was given a general consent form at the beginning of the experiment and a hypnosis consent form after the second VCI. The wording in the relaxation and hypnosis consent forms was as similar as possible. In addition, the hypnosis and relaxation consent forms which were given at the beginning of the experiment incorporated 
the general consent form required by the University (see Appendix $C$ for all consent forms).

\section{Procedure}

All the subjects were recruited for an imagery experiment so that they did not expect "hypnosis" or relaxation until the specified moment in each condition. However, at the end of the experiment there was a full disclosure of the purpose of the study (see Appendix E for the debriefing procedure). The subjects were asked not to discuss the experiment with anyone and were told that they may have their susceptibility scores if interested. The subjects were also assured of confidentiality throughout the experiment, and all the information was coded to ensure this.

Part 1

Volunteers were solicited from psychology courses. The Health Status Questionnaire was given in order to determine the suitability of the volunteers. Suitable volunteers were randomly assigned to one of four groups (each of these groups was broken down further into 3 or 4 subjects per administration). Finally, the subjects were called and appointments were set.

Part 2 (Please see diagram, Appendix A, for clarification)

Group_1. After being introduced to the imagery experiment, the subjects were informed the study also involved hypnosis, and that any of them could leave and 
still receive credit for the study. The hypnosis consent forms were passed out, signed, and collected. The waking instructions for the VCI and the VCI were given, and then the "hypnosis" and the hypnotic instructions for the VCI and the VCI were given. Finally, the HGSHS-A was administered. The subjects were debriefed.

Grouㅁㄹ. After being introduced to the imagery experiment, the subjects were asked to read and fill out the general consent forms. These forms were signed and collected. Next, the waking imagination instructions for the VCI and the VCI were given. Then, it was announced that the second part of the experiment involved hypnosis, and that anyone could leave and still receive credit for the experiment. The hypnosis consent forms were read, signed, and collected. Then the subjects were given the "hypnosis" and hypnotic instructions for the VCI and the second VCI. Finally, the HGSHS-A was administered. The subjects were debriefed.

Gro으묘. Af. After being introduced to the imagery experiment, the subjects were informed the study also involved a relaxation exercise and were told that anyone could leave and still receive credit for the experiment. The relaxation consent forms were passed out, signed, and collected. The waking imagination instructions for the VCI and the VCI were given. The subjects were relaxed and were 
given the "relaxation" instructions for the VCI and the second VCI. After this, they were told that the study also involved a hypnotic susceptibility test, and that anyone could leave and still receive credit for the experiment. The hypnosis consent forms were passed out, signed and collected. Finally, the HGSHS-A was administered, and the subjects were debriefed.

Groung-4. After being introduced to the imagery experiment, the subjects were asked to read and fill out the general consent forms. Then, these forms were signed and collected. The waking instructions for the VCI and the VCI were given. Then, the SQ4R passage was given, followed by the waking instruction for the VCI and the second VCI. Next, the subjects were told that the study also involved a hypnotic susceptibility test, and that anyone could leave and still receive credit for the experiment. The hypnosis consent forms were passed out, signed, and collected. Finally the HGSHS-A was administered, and the subjects were debriefed (see Appendix $C$ for the consent forms and Appendix E for the exact procedures used). 


\section{RESULTS AND DISCUSSION}

The main dependent variable in this experiment was each subject's VCI scores. The VCI test was given twice to each subject in each of the four groups. The highest possible total on each of the two VCI tests was 55; the lowest was 11 . This range was different from the coe et al. study (1980) (i.e., their range was 15 to 75 ) because the first four questions in each VCI was used as a rehearsal and were not included in the present data analyses. A high score on the VCI indicated the subject frequently produced vivid visual images; a low score indicated the subject did not frequently produce vivid visual images.

The mean scores for the first and second administrations of the VCI for each of the four groups (using only the subjects who scored 4 or above on the HGSHS-A) are shown in Figure 1.

Although in Figure 1 it appears that the subjects' VCI scores on the first administration differed across the four groups, a Kruskal Wallis one-way analysis of variance by ranks (with correction for tied ranks) revealed that these differences were not statistically significant $\left(\underline{H}_{\mathcal{C}}=3.35\right.$, $\underline{d f}=3 ; \underline{D}>0.30)$. [Since the VCI scale cannot be con- 


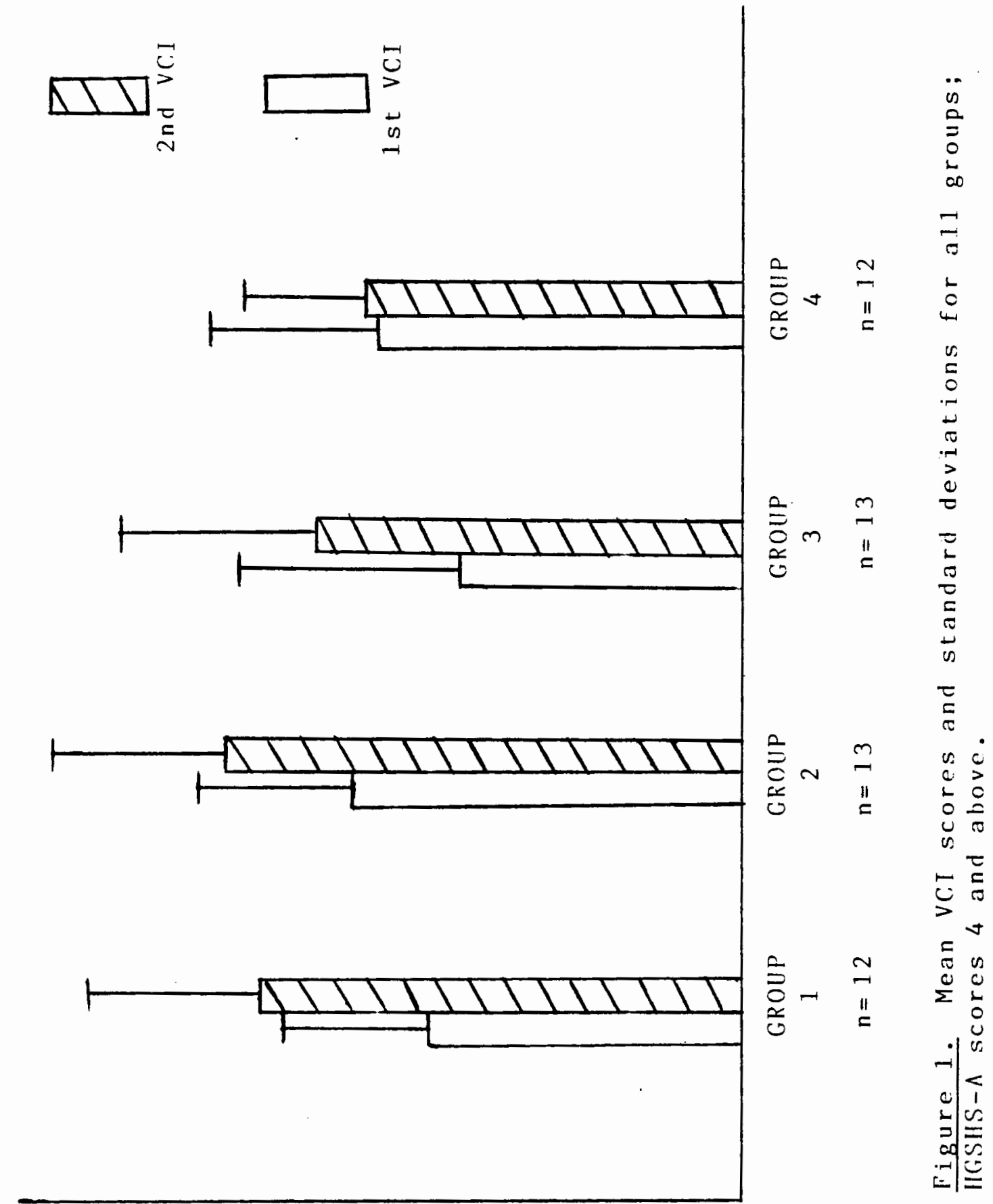

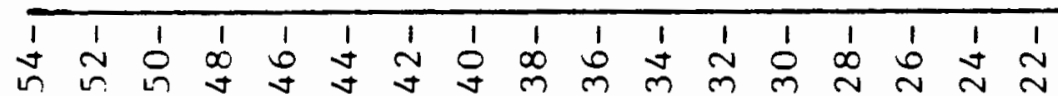


sidered a ratio scale, non-parametric statistics were used]. Figure 1 also seemed to indicate that the subjects' VCI scores on the second administration differed across the four groups, but a second Kruskal Wallis ANOVA (corrected for tied ranks) revealed these differences were also not statistically significant $\left(\underline{H}_{\underline{c}}=4.36\right.$, df $=3$, $\left.p>0.20\right)$.

However, the differences between the VCI scores on the first and second administrations within Groups 1, 2, and 3 were significant. This was statistically confirmed by three Wilcoxon matched-pairs signed-ranks tests. In Group 1 (in which the subjects were expecting hypnosis during the first, waking VCI) the scores for the second VCI $(X=43.75)$ were significantly greater than the scores for the first VCI $(\boldsymbol{X}=35.33: \underline{t}=2, \underline{p}<0.005)$. In Group 2 (in which the subjects were not expecting hypnosis during the first, waking VCI) the scores for the second VCI $(X=45.15)$ were significantly greater than the scores for the first VCI $(\mathbb{X}=39.69: I=8, \underline{p}<0.005)$. Finally, in Group 3 (in which the subjects were expecting relaxation during the first VCI) the scores for the second VCI $(X=41.39)$ were also significantly greater than the scores for the first VCI $(\boldsymbol{X}=34.31$ : $I=0, \mathrm{D}<0.005)$.

The VCI scores between the first and second administrations within Group 4 did not appear to differ. A fourth Wilcoxon matched-pairs signed-ranks test was used to confirm this. In Group 4 (in which the subjects did not expect 
anything during the first or second VCI) the second VCI scores $(X=39.67)$ were not statistically greater than the first VCI scores $(\boldsymbol{X}=38.25: I=19, \mathrm{p}>0.05)$.

\section{Qutcome of the Predictions}

\section{Prediction 1}

This prediction dealt with the view that subjects who are expecting hypnosis (Group 1) may "hold back" on their first VCI in order to do much better on their second. If this occurred, then the first (Waking) VCI scores in Group 1 (expect-hypnosis) would be significantly lower than the first (Waking) VCI scores in Groups 2 and 4 (groups in which the subjects did not expect hypnosis or relaxation during their first VCI).

The present results do not indicate any significant "holding back" pattern for these groups. The scores for the first VCI were relatively equal across the four groups; although there was an insignificant difference between the first VCI scores in the three groups (1, 2, and 4) which did fit the "holding back" pattern: In Group 1 scores were lower $(X=35.33)$ than Group $2(X=39.69)$ and Group 4 $(\underline{X}=38.25$ ) suggesting, that to some extent, the subjects in Group 1 may have anticipated being hypnotized and "held back" on their first VCI in order to excel under "hypnosis." 


\section{Prediction 2}

The second of the "holding back" predictions was that subjects who expected hypnosis during their first (Waking) VCI (Group 1) would "hold back" more than subjects who expected relaxation during the first (Waking) VCI (Group 3). However, those subjects who expected relaxation would "hold back" on their first VCI to some extent, and their scores would be significantly lower than the subjects who did not expect either hypnosis or relaxation during their first VCI (Groups 2 and 4). This would have been indicated by the first VCI scores in Group 3 being significantly greater than the first VCI scores in Group 1 but significantly lower than the first VCI scores in Groups 2 and 4.

However, the results show this was not the case for this sample because there was no significant difference between the first VCI scores across the four groups (see Figure 1). Again, however, there was a trend toward the "holding back" pattern for Group 3 (the expect-relaxation group): the first VCI scores for Group 3 were lower $(X=34.31)$ than for Groups $2(X=39.69)$ and $4(X=38.25)$ and were also lower than the first VCI scores for Group 1 $(\mathbb{Z}=35.33)$ (see Figure 1). Although these differences were not large or significant, this indicates that Group 3 may have anticipated being relaxed and "held back" on their first VCI scores at least as much as the subjects in Group 1. This suggest that expectation in general, not just 
expectation of hypnosis, may cause, at least, a slight "holding back" effect.

Scharf and Zamansky (1963) and Zamansky et al. (1964) found that their subjects "held back" on a waking exercise in order to do better on a hypnotic exercise. However, Coe et al. (1980) did not find the "holding back" pattern in their study.

\section{Prediction 3}

The last of the "holding back" predictions was that the expectation of hypnosis (Group 1) would be more powerful than the expectation of relaxation (Group 3). This would have been indicated if the difference between the scores for the two administrations of the VCI in Group 1 was significantly greater than the difference between the scores for the two administrations of the VCI in Group 3. Many researchers like Orne (1959, 1967) and Lazarus (1973) consider the expectation of hypnosis very powerful; whereas, the expectation of relaxation should not have the same effect. This prediction would have also been indicated because Hamberger and Lohr (1980) and the study found within that article (Rehm et al., 1974) found that relaxationdid not increase vividness of visual imagery but may even decrease vividness; therefore, any increases in VCI scores would be due to expectation because a relaxation exercise was used for all three experimental groups. These factors 
would cause subjects to "hold back" more in Group 1 (expecthypnosis) than in Group 3 (expect-relaxation) and, consequently, the subjects in Group 1 would show a significantly greater leap in their vividness scores than the subjects in Group 3 by the time the second VCI was given.

This prediction was not supported by the present results. A Mann-Whitney U-test on the difference scores was completed, and the differences between the first and second VCI scores in Group 1, as compared to Group 3, were not found to be significant $\underline{U}=65.6, \underline{D}>0.05$. These results indicate that expectation of relaxation in Group 3 increased the vividness of visual imagery as well as expectation of hypnosis did in Group 1: the opposite of what was predicted by Hamberger and Lohr (1980), Orne (1959, 1967), or Lazarus (1973). This situation could have occurred because expectation of relaxation increased the vividness of imagery in Group 3 or because the relaxation exercise itself increased the vividness of visual imagery because it was not comprehensive enough to decrease the vividness. Jacobson (1957) found that visual imagery is not hindered by relaxation unless the eyes are relaxed; therefore, the subjects in the present study may not have had their eye muscles relaxed enough to decrease visual imagery because the relaxation technique in this study did not concentrate on relaxation of 
the eyes of in particular and was only approximately 8 minutes in length.

Since the relaxation technique used in this study may have increased the vividness of visual imagery when correctly labeled, it will be difficult to determine whether the difference between the first and second VCI scores in Groups 1 and 2 (both groups expected hypnosis by the time the second VCI was administered) were due to expectation of hypnosis or the relaxation technique or some combination of the two.

\section{Prediction 4}

This prediction dealt with the Behavioristic view put forth by Sarbin and Coe (1972) that the mere mention of the word hypnosis would increase expectation and, thereby, increase the vividness of visual imagery from the instant that the word hypnosis was mentioned. Hence, the scores for the first VCI in Group 1 (expect-hypnosis) would be significantly greater than the first VCI scores in any other group, and the scores for the second VCI in Group 2 (in which the subjects did not know about the "hypnosis" until just prior to taking the second VCI) would be about equal to the scores for the second VCI in Group 1 because hypnosis was mentioned at the beginning of the session in Group 1 and the middle of the session in Group 2 (see Appendix E for the procedures used in each group). 
This prediction was not found to be an accurate description of the present sample distribution since all the scores for the first VCI across the four groups were relatively equal, and all the scores for the second VCI across the four groups were relatively equal. Therefore, the mention of the word hypnosis did not automatically increase the vividness of visual Imagery in any significant manner.

However, in the group of subjects who were not included in the overall data analysis (i.e., subjects who scored $0-3$ on the HGSHS-A) the subjects in Group 1 had very high scores on the first VCI $(X=42.00)$ and only raised these scores by an average of 5.3 points on the second VCI $(X=47.33)$. This may indicate some reaction to the mention of hypnosis by the subjects who had low hypnotic susceptibility, but there were only 3 subjects in Group 1 and no subjects in Group 2 (in the sample of low scorers); therefore, the questions that this stirs can only be addressed in future research. (See Appendix G for VCI score pattern and Appendix $F$ for individual subjects data).

\section{Predicton 5}

This prediction dealt with the effect of using the same imagery test twice for each subject. It stated that if this practice effect was the main reason for the difference between the first and second VCI scores in all four groups, then the difference between the scores for the first and 
second VCI tests in Groups 1, 2, and 3 would not be significantly greater than the difference between the scores for the first and second VCI tests in Group 4.

To test this prediction statistically, a Kruskal Wallis ANOVA was performed on the difference scores between the first and second VCI administration for all four groups. This test confirmed there were significant differences between the four groups: $\underline{H}_{\underline{C}}=9.50, \underline{d f}=3 ; \underline{D}<0.05$.

To further test which groups differed, six MannWhitney U-test were also completed. When Group 1 (expecthypnosis during the first VCI) was compared to Group 4 (no expectations set), it was found that the differences between the first and second VCI scores in Group 1 were significantly greater than the differences found in Group 4: $\underline{U}=23, \underline{p}<0.01$. When Group 2 (expect-hypnosis only during the second VCI) was compared to Group 4, it was found that the differences between the first and second VCI scores in Group 2 were significantly greater than the differences found in Group 4: $\underline{U}=43$, $\underline{p}<0.05$. When Group 3 (expect relaxation during the first VCI) was compared to Group 4, it was found that the differences between the first and second VCI scores in Group 3 were significantly greater than the differences found in Group 4: $\underline{U}=37, \underline{p}<0.025$. However, all comparisons between the three experimental groups were not found to be significant (U's > 54.5, D's >0.05). 
The above findings indicate that a practice effect was not a major factor working in this experiment because the differences between the first and second VCI scores in the three experimental groups $(1,2$, and 3 ) were significantly greater than the difference between the first and second VCI scores in the control group (Group 4). In fact Group 4 was the only group in which the difference between the first and second VCI scores was not significant (first VCI $\mathbb{Z}=38.25$; second VCI $X=39.87$ ): $75 \%$ of the subjects in this group had a 3 (or less) point difference between their two VCI scores.

\section{Prediction 6}

This prediction dealt with the Phenomenological view put forth by Tart (in Fromm \& Shor, 1972) and found in Andreychuk and Skriver (1975), and Knox and Shum (1977) that highly susceptible individuals can slip into a hypnotic state without any formal induction. If this is the case, then we might examine only highly susceptible subjects for evidence of slippage (i.e. subjects who scored 9-12 on the HGSHS-A). In addition because Group 4 was the only group which did not have any expectations experimentally set, it is the only group which can be examined for hypnotic "slippage." If hypnotic "slippage" did occur, then the scores for the second VCI would be significantly greater than the scores for the first VCI for highly susceptible 
subjects in Group 4, and the scores for the second VCI in Group 4 of the medium susceptible subjects (HGSHS-A 4-8) would not greatly differ from their first VCI scores (indicating that no factor other than hypnotic "slippage" could be raising the second VCI scores for the highly susceptible subjects).

In the present study the highly susceptible subjects did score higher on their second VCI test $(\underline{X}=48.5)$ than they did on their first VCI text $(X=43.5)$, and the second VCI scores for the medium susceptible subjects in Group 4 were not found to be significantly different (by a Wilcoxon test) from their first VCI scores $(I=19$, $p>0.05)$. However, there were only two highly susceptible subjects in Group 4; therefore, this prediction cannot be answered with any confidence.

Still, since hypnotic slippage is an individual characteristic and may get lost in group analysis, the two subjects who did fit into this category should be examined. One of the subjects had experienced hypnosis once before in a stage show; his score on the second VCI did not change at all from his first score (45) indicating that he was not affected by a practice effect, nor did he "slip" into a hypnotic state. The other subject who meditated regularly raised his first VCI of 42 to a score of 52 on the second. This suggests that this subject may have "slipped" into a hypnotic state because the first subject who achieved the 
same susceptibility score on the HGSHS-A (9) as this second subject and also had prior experience with hypnotic states, did not improve on his second VCI test (see Appendix F). Since the 10 point change between the two VCI tests for the second subject cannot be attributed to a practice effect or expectation nor can it be attributed to hypnotic experience or the differences in the hypnotic susceptibility scores of these two subjects, it is likely, that this (second) subject "slipped" into a hypnotic state. However, it is difficult to "prove" this prediction since the subjective experience of hypnosis is said to be very different for every subject (Fromm \& Shor, 1972). In the present sample a more even number of subjects were needed in each susceptibility level to further test the high (and low) susceptibility groups.

If hypnotic "slippage" did occur in Group 4, it is possible that some of the other highly susceptible subjects in the other three groups also "slipped" into a hypnotic state. Since there is no way to test for hypnotic "slippage" in the other three groups, it is important to examine how many subjects could have "slipped." In Group 1 $41.47 \%$ of its subjects were highly susceptible and could have "slipped" into a hypnotic state. In Group $223.08 \%$ of its subjects were highly susceptible (i.e., scored 9 or higher on the HGSHS-A), and in Group $315.39 \%$ of its subjects were highly susceptible. Therefore, Group 1 is the 
only group in which the subjects' scores could have been significantly affected by hypnotic "slippage." Hypnotic "slippagen may have contributed to the difference between the first and second VCI scores in Group 1.

\section{The Sample}

Although the subjects were randomly placed into the four groups, some differences occurred in the sample (including the low scorers: $0-3$ on the HGSHS-A) which may have affected the outcome: (1) Women scored significantly lower than did men $\left(X^{2}=5.66, d \underline{f}=1 ; \underline{p}<0.02\right)$; and (2) subjects 30-49 years of age scored significantly lower on the HGSHS-A than did subjects 18-29 years of age $\left(X^{2}=4.22\right.$, $\underline{\mathrm{df}}=1 ; \mathrm{P}(0.05)$. [The preceding calculations used all data available including the low HGSHS-A scorers: $0-3$, and the three susceptibility levels were collapsed into two levels: for these calculations $0-6$ on the HGSHS-A was considered low and 7-12 was considered high.] Thus, there were two kinds of people who tended to have more vivid visual images: (1) men, and (2) persons less than 30 years of age.

Since a greater degree of hypnotic susceptibility has been shown to correlate with a greater ability to see more vivid visual images regardless of the state of consciousness (Diamond \& Taft, 1975; Wagman \& Stewart, 1974; Shor et al., 1966; Spanos et al., 1976; Spanos et al, 1973; Sutcliffe et 
al., 1970; and Palmer \& Field, 1968), results in groups with a greater percentage of "low" or "high" scorers (on the HGSHS-A) may have been influenced by this variable. Groups 2 and 4 had high percentages ${ }^{2}$ of subjects who were better able to produce vivid visual images (Group 2: $53.87 \% \mathrm{men}$, 69.23\% persons 19-29 years old, and 46.15\% men 19-29 years old; and Group 4: $66.67 \%$ men, $91.67 \%$ persons 19-29 years old, and $66.67 \%$ men 19-29 years old). Group 1 also held a relatively high percentage of subjects who were better able to produce vivid visual images $(50.00 \% \mathrm{men}, 66.67 \%$ persons 19-29 years old, and 33.34\% men 19-29 years old), but Group 3 had a low percentage of subjects who were better able to produce vivid visual images (15.38\% men, $69.23 \%$ persons 1929 years old and $7.69 \%$ men $18-29$ years old). Please see Table II.

These differences in susceptibility level for each group could have affected the results accordingly: (1) In Group 1 (relatively high percentage of "high" HGSHS-A scorers) the first VCI scores may have been artificially elevated, and this may have made the slight "holding back" trend found seem less prominent; (2) In Group 2 (high percentage of "high" scorers) the scores for the first VCI also

2 The following percentages do not include the data from low $(0-3)$ HGSHS-A scorers because these subjects did not affect the analyses discussed. 


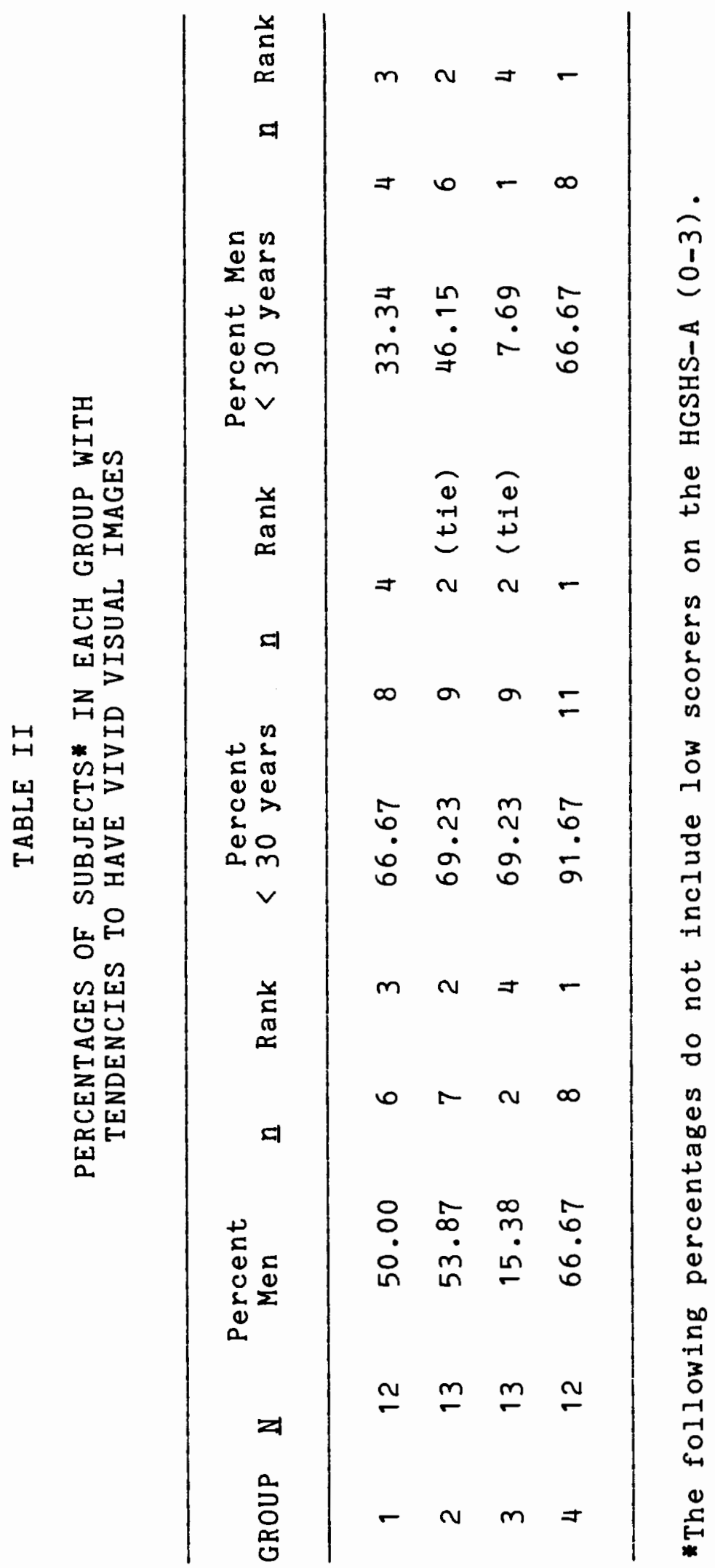


could have been artificially elevated which may have made the "holding back" trend found seem more prominent (since the "holding back" prediction is a comparison of the first VCI scores). However, since both groups ( 1 and 2 ) may have been elevated, the difference caused by the susceptibility correlation probably would not have changed the ratio between these two groups' VCI scores in any significant manner; (3) In Group 3 (low percentage of "high" scorers) the scores on the first VCI could have been artificially suppressed, and this could have caused the "holding back" trend found for relaxation to appear more prominent; and (4) In Group 4 (high percentage of "high" scorers) the first VCI scores could have been artificially elevated which also may have played a part in the overall "holding back" trend found.

Since Group 4 was not complicated by the expectation of hypnosis or relaxation, the difference between the two VCI scores in this group can be examined to see if the sample characteristics, mentioned above, affected the change in VCI scores from the first administration to the second. Upon examination, there was no significant difference between the VCI scores for the two administrations in Group 4 (see Figure 1). This suggests that the majority of subjects in this group did not greatly increase their ability to produce vivid visual images even though Group 4 had the largest percentages of subjects who were more likely to 
raise their second VCI scores because of their natural ability to produce vivid images. Hence, the differences between the first and second VCI scores in the other three groups were probably not caused by the high percentages of subjects who had higher HGSHS-A scores in some of these groups. Although, these sample variables and the experimental variables (i.e., expectation of relaxation or expectation of hypnosis) could have interacted to change the effect the experiment was supposed to have had on the subjects.

Therefore, the non-significant "holding back" trend found in the present experiment was probably mostly due to sample characteristics. In Group 1 the scores on the first VCI seemed lower because scores in Groups 2 and 4 were artificially elevated; the scores in Group 1 were also elevated but not the same extent. Also, in Group 3 the scores on the first VCI seemed lower because the scores in groups 1, 2, and 4 were artificially elevated and the scores in Group 3 were artificially suppressed. This greatly weakens findings that the "holding back" trend in the relaxation group (Group 3) was greater than the "holding back" trend in the "hypnosis" group (Group 1), and that this was because the subjects were affected by the expectation of relaxation at least as much as they were affected by the expectation of hypnosis. Consequently, the only "holding 
back" trend left, after taking into account the sample characteristics, is a very slight trend to this effect in Group 1 (expect-hypnosis).

\section{The Relaxation Technique Used}

As mentioned previously, there is the possibility that the relaxation technique used in this study may have increased vividness of visual imagery instead of not increasing it, or even decreasing it, as expected (Hamberger \& Lohr, 1980; Rehm et al., cited in Hamberger \& Lohr). This makes it difficult to differentiate the effect of expectation of relaxation or hypnosis from the effect of the relaxation technique itself. However, one factor does seem to shed some light on this dilemma. The sample included in the main analyses (using subjects scoring 4 or above on the HGSHS-A) seemed to fall into two categories: (1) subjects who changed a great deal between the two VCI administrations (i.e., a difference of 7 or more points), indicating either a reaction to the relaxation technique or expectation, and (2) subjects who changed very little between the two VCI test (i.e., a difference of 3 or less points), indicating the expected and predicted reaction to the relaxation technique and no reaction to the experimental conditions.

The groups which set the expectation at the beginning of the experiment (Groups 1 and 3) had a greater percentage of subjects with 7 or more points difference between their 
two VCI scores (Group 1: $83.33 \%$ and Group 3: 46.17\%), than did Group 2, in which expectation of hypnosis was set after the subjects had taken their first VCI $30.77 \%$ had $7 \leq$ difference) indicating that expectation accounted for some of the variance of scores in Groups 1 and 2. In addition, Group 1 (expect-hypnosis) had a much greater percentage of subjects with a 7 or greater point difference (83.33\%) than did Group 3 (expect-relaxation) ( $46.17 \%$ ), indicating that expectation of hypnosis was more powerful in increasing vividness of visual imagery than was the expectation of relaxation. In fact all the subjects in Group 1 either scored a 7 or greater point difference or scored a point difference of 3 or less (16.67\%). Consequently, expectation of hypnosis alone seemed to increase vividness of visual imagery above and beyond general expectations (i.e., expectations of relaxation in Group 3) and the effect of the relaxation technique by itself.

\section{Lazarus (1973)}

Although the preceding results lend some support to the Lazarus findings that expectations of hypnosis were an important factor in enhancing vividness of visual imagery, the major results of the present study do not support his general findings. Lazarus found that his clients who expected to be hypnotized but were given a relaxation exer- 
cise labeled "hypnosis" had more vivid visual imagery and improved more in therapy than did his clients who expected to be hypnotized but were given the same relaxation exercise correctly labeled. These findings indicated to Lazarus that expectation of hypnosis was an extremely important factor in hypnosis because relaxation labeled "hypnosis" seemed to produce the same effect as hypnosis, and this relaxation technique labeled correctly did not produce as strong an effect.

The present study found that the subjects who were given a relaxation exercise (the same exercise used in the Lazarus study) labeled "hypnosis" did not have significantly greater vividness of imagery (VCI scores) than did the subjects who were given the relaxation technique correctly labeled. Both these groups significantly improved their vividness of visual imagery from the first VCI administration. These findings suggest that expectation of relaxation and/or relaxation, in its own right produce some of the same effects that hypnosis has been found to produce. In addition, since Jacobson (1957) found that relaxation exercises enhance visual imagery unless the eye muscles are specifically relaxed, and the relaxation exercise used in both the Lazarus study and the present study did not specifically focus on the eyes, the conclusions that Lazarus came to come into question. First of all, if the relaxation exercise used in the present study enhanced vividness of visual 
imagery, yet the same exercise did not enhance visual imagery in the Lazarus study, then it is possible that imagery was enhanced by the relaxation exercise in the Lazarus study, but it was not adequately measured by him. First, Lazarus knew which group ("hypnosis" or relaxation) each of his clients were in, and he was also the one who decided which clients had more vivid visual images; therefore, this measurement of imagery cannot be considered unbiased. Second, a great deal of the expectations in the Lazarus study were probably due to the client-therapist relationship rather than whether or not the clients received the relaxation exercise labeled "hypnosis" or relaxation. This could be because the clients who received the correctly labeled relaxation exercise did not receive what they specifically requested, paid for, and felt they needed to solve their problems which were serious enough to cause them to seek professional help. It is no wonder these clients did not improve or see more vivid images. Hence, Lazarus' findings may have been more indicative of the emotional stake both his clients and he had in the outcome rather than the true nature of expectations outside the therapeutic setting. 


\section{Coe, St. Jean, and Burger (1980)}

Both Coe et al. (1980) and the present study found no significant "holding back" effect, although the present study did find a very slight indication of the "holding back" effect in the expect-hypnosis group (Group 1). Coe et al. also found that hypnosis enhances vividness of visual imagery. The present results indicated that expectation of hypnosis and expectation of relaxation, and maybe even the relaxation technique itself, significantly enhanced vividness of visual imagery. In addition, the present results indicate that the enhancement of the vividness of visual imagery was not due to a practice effect. This suggests that the similar findings of Coe et al. (1980) were also not due to a practice effect.

Finally, the present study found that if trust was fostered in the subjects, their hypnotic susceptibility increased. (All the subjects who participated in the experiment are included in the following calculations). The subjects in Group 2 were the only persons who experienced a waking VCI before they were told they would be hypnotized and take another VCI. Because these people had an idea of what being "hypnotized" was like (it was actually a relaxation technique), and they knew exactly what they were expected to do under "hypnosis," they had the highest mean HGSHS-A score, 7.46, of the four groups, and no subject in 
this group scored less than 4 on the HGSHS-A (i.e., everyone tested in this group met the criterion to be included in the main data analyses). Group l's subjects, however, were informed about the hypnosis at the beginning of the experiment, then they took the first VCI and were "hypnotized." Again, these subjects knew that the only activity they were going to be involved in during hypnosis was their second VCI. Hence, this group also thought they had experienced "hypnosis" before they took the HGSHS-A, and they had the second highest mean HGSHS-A score, 7.00 ( $20 \%$ of these subjects had a score too low to be included in the main data analyses.) (This high percentage of low scorers could have been due to being informed about the "hypnosis" at the beginning of the experiment because the subjects may have felt deceived, because they had volunteered for an imagination experiment, but, the moment the session began, were told they were to be hypnotized; there was not enough time to build trust). In Group 3 the subjects had no experience with hypnosis (in most cases), although they participated in the same relaxation exercise that Groups 1 and 2 experienced before they took the HGSHS-A; consequently, they did not know what being "hypnotized" was like, nor did they know what they were expected to do under "hypnosis," and they had a lower mean HGSHS-A score, 5.88. In addition $18.8 \%$ of the subjects in this group scored too low to be included in the main data analyses. Finally, the subjects in Group 4 did 
not experience "hypnosis" or relaxation (a related experience) before they took the HGSHS-A, and, again their scores were affected accordingly: Mean HGSHS-A score 5.12, and 29\% of these people's scores were too low to be included in the main data analyses.

In the light of the preceding information, the mean VCI scores of the second experiment of the Coe et al. study also suggest that an atmosphere of trust increased hypnotic susceptibility (shown by the enhancement of visual imagery, VCI scores, which has been found to be positively correlated with hypnotic susceptibility). (Since coe et al. gave their hypnotic susceptibility measure at different times: either in the middle of the session for the Hypnosis-only condition, or at the end of the session for the Waking-only condition, these hypnotic susceptibility measures would not reveal the differences between each condition which would affect the atmosphere of trust. In addition, since coe et al.'s second experiment used a real hypnotic induction, the effect of an actual hypnotic state complicates using hypnotic susceptibility scores for the present purpose.) In their second experiment they enlisted subjects for an "imagination" study, and only after the subjects arrived, were they told that the experiment also involved hypnosis. In the Waking-only condition the subjects took the waking VCI before they had any knowledge that they were to be 
hypnotized, but the subjects in the Hypnosis-only condition were hypnotized at the beginning of the experiment (after giving their consent to be hypnotized), and then they took their first and only VCI under hypnosis. Consequently, the subjects in this second condition were not sure what they would be asked to do while hypnotized nor how being hypnotized would make them feel. Hence, it is no surprise that there was virtually no difference between the VCI means of these two conditions (across all three susceptibility levels) because the subjects in the second condition (Hypnosis-only) were experiencing the VCI and hypnosis for the first time. With the newness and tension of the situation, it is not likely that these subjects' VCI scores would be higher than the comparatively safe (at least while the VCI was given) Waking-Only condition. (Coe et al.'s first experiment could not be examined here because all of those subjects were hypnotized prior to being assigned to the experimental conditions; therefore, they knew hypnosis was involved and had already experienced it by the time they participated in the main experimental conditions.)

\section{Conclusions}

The present results do not give a clear picture of the part that expectation plays in hypnosis, because, after the experiment was already in progress, it was discovered that relaxation could enhance visual imagery (Jacobson, 1957). 
Consequently, it is difficult to determine whether the change in the subjects' vividness of visual imagery was due to expectation (of hypnosis or relaxation) or the relaxation technique itself. An analysis which split the subjects into those whose VCI scores changed by 7 (or more) or 3 (or less), however, suggests that expectation of hypnosis did enhance the vividness of visual imagery more than the expectation of relaxation which, in turn, seemed to enhance the vividness of visual imagery more than the relaxation technique used.

It is also possible that expectation (of hypnosis or relaxation) or the relaxation exercise could have been the mediating step between a normal waking state and a hypnotic state. Ludwig (1966) proposed that there was a critical level of stimulation which keeps an individual in a normal state of consciousness, and if this stimulation level is either increased or decreased beyond this critical range, the individual can readily move into a hypnotic state. Malott's findings (1984) supported this proposal. Malott had his subjects either pedal stationary bicycles, listen to a verbal induction, participate in a combination of the two, or listen to a traditional relaxation induction. 3 Malott

3 The Stanford Hypnotic Susceptibility Scale was used as the traditional relaxation induction. 
found that the group who participated in the combination verbal induction and pedaling and the group who listened to the traditional relaxation induction had almost identical mean SHSS scores, and both of these means were significantly higher than the other two groups (i.e., pedaling or the verbal induction alone). He theorized that

the combination of verbal active-alert induction and bicycle pedaling appears to represent a level of stimulation above the hypothetical range.... Conversely, the traditional relaxation induction can be regarded as a technique for reducing stimulation to levels below that range (Malott, 1984, p. 248).

If the present results are examined in the reflection of the preceding studies (Ludwig, 1966; Malott, 1984), the relaxation technique used in the present study could have lowered the stimulation level of the subjects below the critical range, and the subjects could have actually become hypnotized. In addition, the expectation of the relaxation or hypnosis could have caused the lowering of the stimulation level causing a "slippage" into hypnosis (and/or the breakdown of the Generalized Reality-Orientation discussed on pages 12 and 13).

The present results may also shed some light on Behaviorist vs. Phenomenologist views of hypnosis. The two groups may have such opposing views because they tend to study different types of people. When the subjects used in the main data analyses (HGSHS-A scores 4-12) were examined, it was found that they did not automatically respond to the 
word hypnosis (one of the Behavioristic predictions; prediction 4). Yet, the subjects who were not included in the main analysis because their susceptiblity scores were too low (1.e., 0-3) did seem to respond to the word hypnosis. As stated before, Behavioral researchers tend to use these inexperienced subjects who have no great hypnotic ability. The Phenomenologists, on the other hand, tend to study individuals with proven hypnotic ability who may not have the same expectations of hypnosis. Further research should explore the interaction that expectation and level of susceptibility may create.

In addition these two viewpoints may be examining two different stages of hypnosis while trying to explain the entire process. The expectation of hypnosis and the social situation in which the subjects are placed (the process that the Behaviorists examine) may be a mediating step, the needed change in stimulation, proposed by Ludwig (1966), required to move the individual to a different state of consciousness (which is the major process examined by the Phenomenologists).

The present results may also indicate that it is not the prestige of the situation that causes the subjects to "act" hypnotized (as the Behaviorists suggest), but it may be the faith that the subjects have in the experimenter, and the trust that is nurtured in the experiment prior to the hypnotic experience that allows the subjects to become 
hypnotized. This trust may allow the subjects to relax enough to lower stimulation below the level required to keep them in a normal state of wakefulness.

The results also suggest that expectations need to be examined more in future research because there is some indication that expectation of hypnosis does affect the subjects' subsequent hypnotic performance. However, future research should focus on expectation; it should not be something that is studied in passing. If the present experiment is ever replicated, a different, more extensive relaxation technique should be used (or compared to the relaxation exercise used in the present study). An in depth examination of how relaxation differs from hypnosis is also needed. Finally, the results suggest that clinicians using hypnosis should focus on making their clients feel secure enough to allow themselves to become hypnotized.

\section{Summary of Findings}

Taking into consideration the uneven distribution of subject characteristics in the sample and the unexpected effect of the relaxation technique used, the present study found (1) A significant difference between the first and second VCI scores in the expect-hypnosis group, the do-notexpect-hypnosis group, and the expect-relaxation group, indicating that vividness of visual imagery was signifi- 
cantly enhanced by all three experimental conditions. There was also non-significant evidence that the expectation of relaxation increased the enhancement of imagery above the level of the relaxation technique alone, and, finally, that the expectation of hypnosis increased the enhancement of imagery above the level of enhancement induced by the expectation of relaxation. (2) No significant "holding back" effect was found. (3) No significant evidence of subjects reacting to the mere mention of the word hypnosis was found. (4) A practice effect was found to be an insufficient reason for the results in the three experimental groups. (5) A slight indication of hypnotic "slippage" was found, but there were too few subjects in the high susceptibility group to be conclusive. (6) Finally, feelings of security seemed to increase hypnotic susceptibility. 
REFERENCES

Andreychuk, T., \& Skriver, C. (1975). Hypnosis and biofeedback in the treatment of migraine headaches. Inter $=$ national Journal of Clinical and Experimental Hypnosis, 23, $172-183$.

Bentler, P. M., \& Hilgard, E. R. (1963). A comparison of group and individual induction of hypnosis with selfscoring and observer-scoring. International Journal of Clinical and Experimental Hypnosis, 11, 49-54.

Brown, H. A., \& Krasner, L. (1969). The role of subject expectancies in hypnosis. International Journal of Clinical and Experimental Hypnosis, 17, 180-188.

Cautela, J. R. (1975). The use of covert conditioning in

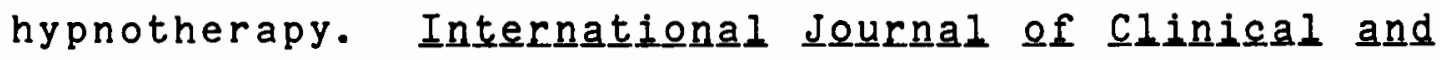
Experimental Hypnosis, 23, 15-27. Coe, W. C., St. Jean, R. L., \& Burger, J. M. (1980). Hypnosis and the enhancement of visual imagery. International Journal of Clinical and Experimental Hyonosis, 28, $225-243$.

Dengrove, E. (1973). The uses of hypnosis and behavior. International Journal of clinical and Experimental Hypnosis, 21, 13-17. 
Diamond, M. J., \& Taft, R. (1975). The role played by ego permissiveness and imagery in hypnotic responsivity. International Journal of clinical and Experimental Hypnosis, 23, 130-138.

Estrabrook, G. H. (1930). A standardized hypnotic technique dictated to a Victrola record. American Journal of Psychology, 42, 115-116.

Fromm, E., \& Shor, R. E. (1972). Underlying theoretical issues: an introduction. In E. Fromm \& R. E. Shor (Eds.), Hypnosis 1 researach deyelopments and perspectives (pp. 3-14). New York: Aldine \& Atherson.

Ham, M. W., \& Spanos, N. P. (1974). Suggested auditory and visual hallucinations in task-motivated and hypnotic subjects. American Journal of Clinical Hypnosis, 17, 94101 .

Hamberger, K., \& Lohr, J. M. (1980). Relationship of relaxation training to the controllability of imagery. Perceptual and Motor Skills, 51, 103-110.

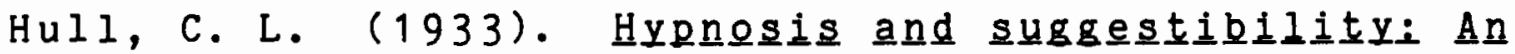
Experimental Approach. New York: Appleton-CenturyCrofts.

Jacobson, E. (1957). Yoㅡㅁ mustㅡ relax. New York: McGrawHill. pp. 165-166.

Knox, V. J., \& Shum, K. (1977). Reduction of cold-pressor pain with acupuncture analgesia in high- and low-hypnotic subjects. Journal of Abnormal Psychology, 86, 639-643. 
Kroger, W. S., \& Felzer, W. D. (1976). Hypnosis and

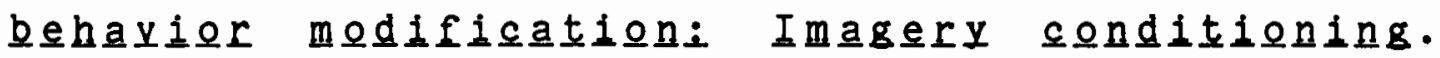
Philadelphia: Lippincott.

Lazarus, A. A. (1971). Behavior therapy and beyond. New York: McGraw-Hill.

Lazarus, A. A. (1973). "Hypnosis" as a facilitator in behavior therapy. International Journal of Clinical and Experimental Hypnosis, 21, 25-31.

Ludwig, A. M. (1966). Altered states of consciousness. Archives of General Psychiatry, 15, 225-234. Malott, J. M. (1984). Active-alert hypnosis replication and extension of previous research. Journal of Abnormal Psychology, 23(2), 246-249.

Marks, D. F. (1973). Visual imagery differences in the recall of pictures. British Journal of Psychholiogy, 64 , $17-24$.

O'Connell, D. N. (1964). An experimental comparison of hypnotic depth measured by self-ratings and by an objective scale. International Journal of clinical and Experimental Hypnosis, 12, 34-46.

Orne, M. T. (1959). The nature of hypnosis: Artifact and essence. Journal of Abnormal and Social Psychology, 58, 277-299. 
Orne, M. T. (1967). What must a satisfactory theory of hypnosis explain? International Journal of Psychiatry, $3,206-211$.

Palmer, R. D. \& Field, P. D. (1968). Visual imagery and

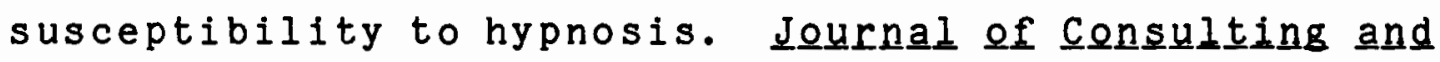
Clinical Psychology, 32, 456-461.

Rehm, L. P., Mattei, M. L., Potts, S., \& Skolnick, M.

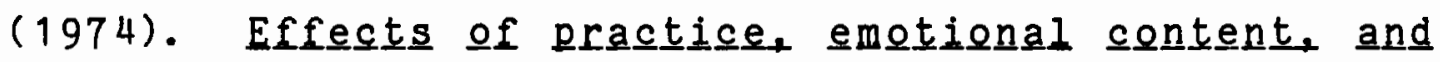

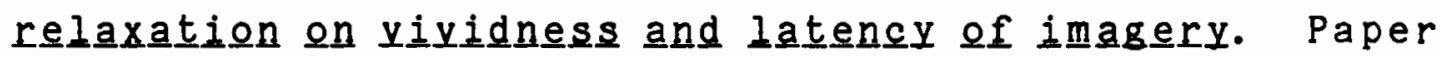
presented at the Eighth Annual Meeting of Behavior Therapy. Chicago, December.

Rossi, A. M., Sturrock, J. B., \& Solomon, P. (1963). Suggested effects on reported imagery sensory deprivation. Perceptual and Motor Skills, 16, 39-45.

Sanders, S. (1969). The effect of hypnosis on visual imagery (Doctoral dissertation, University of Kentucky, 1967), Dissertation Abstracts International, 30, 2936B2937B (University Microfilms No. 69-15, 484).

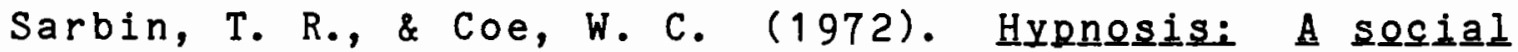
psychological analysis of influence communication. New York: Holt Rinehart \& Winston.

Scharf, B., \& Zamansky, H. S. (1963). Reduction of word recognition threshold under hypnosis. Percenetual angl Motor Skills, 17, 499-510. 
Sheehan, P. W. (1972). Hypnosis and manifestations of 'imagination.' In E. Fromm \& R. E. Shor (Eds.), Hypnosis research deyelopments and perspectiyes (pp. 293-319). New York, Aldine \& Atherson.

Shor, R. E. (1959). Hypnosis and the concept of the generalized reality-orientation. American Jounrnal of Psychotherapy, 13, 582-602.

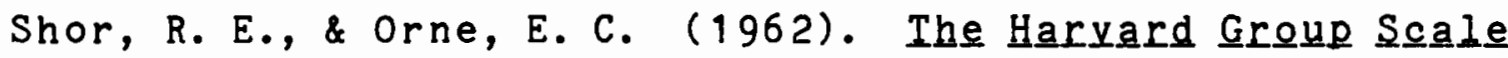

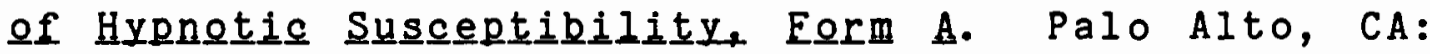
Consulting Psychologists Press.

Shor, R. E., \& Orne, E. C. (1963). Norms on the Harvard Group Scale of Hypnotic Susceptibility, Form A. International Journal of clinical and Experimental Hypnosis, 11, 39-47.

Shor, R. E., Orne, M. T., \& O'Connell, D. N. (1966). Psychological correlates of plateau hypnotizability in a special volunteer sample. Jounrnal of Persongality and Social Psychology, 3, 80-95.

Siebert, A., \& Walter, T. (1983). Student manual to accom= pany understanding human behavior. 4 th edition by J. V. McConnel1. New York: Holt Rinehart \& Winston (pp. 1517).

Spanos, N. P., Churchill, N., \& McPeake, J. D. (1976). Experimental response to auditory and visual hallucination suggestions in hypnotic subjects. Journal of Consulting and clinical Psychology, 44, 729-738. 
Spanos, N. P., Valois, R., Ham, M. W., \& Ham, M. L. (1973). Suggestibility and vividness and control of imagery. International Jounrnal 의 Clinical and Experimental Hyenosis, 21, 305-311.

Stam, H. J., \& Spanos, N. P. (1980). Experimental designs, expectancy effects, and hypnotic analgesia. Journal of Abnormal Psychology, 89, 751-762.

Starker, S. (1974). Effects of hypnotic induction upon visual imagery. Journal of Nervous and Mental Disorders, 159, 433-437.

Stross, L., \& Shervin, H. (1962). Differences in thought organization between hypnosis and waking state: An experimental approach. Bulletin of the Menninger Clinic, 26, $237-247$.

Sutcliffe, J. P., Perry, C. W., \& Sheehan, P. W. (1970). Relation of some aspects of imagery and fantasy to hypnotic susceptibility. Journal of Abnormal Psychology, 76, 279-287.

Tart, C. T. (1972). Measuring the depth of an altered state of consciousness, with particular reference to self-report scales of hypnotic depth. In E. Fromm \& R. E. Shor (Eds.). Hypnosis research developgments and perspectives (pp. 445-493). New York: Aldine \& Atherson. 
Wadden, T. A., \& Flaxman, J. (1981). Hypnosis and weight loss: A preliminary study. International Jounrnal of Clinical and Experimental Hypnosis, 29, 162-173. Wagman, R., \& Stewart, C. G. (1974). Visual imagery and hypnotic susceptibility. Perceptuaㅣ Motor skills, 38 , 815-822.

Weitzenhoffer, A. M., \& Hilgard, E. R. (1959). Sttanferd Hypnotic Susceptibility scale, Forms A and B. Palo Alto, CA: Consulting Psychologists Press. Weitzenhoffer, A. M., \& Hilgard, E. R. (1962). Sitannford

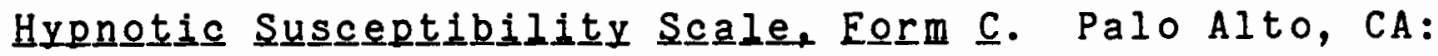
Consulting Psychologists Press. Wolpe, J. (1969). Ihe practices of behavior therapy. Elmsford, NY: Pergamon. Zamansky, H. S., Scharf, B. \& Brightbill, R. (1964). The effect of expectancy for hypnosis on pre-hypnotic performance. Journal of Personality, 32, 236-248. 
Addendum November 17, 1987

CORRECTION REGARDING HYPNOTIC SUSCEPTIBILITY IN COE ET AL. $198 \emptyset$

Subjects in the Waking-First group did score differently on their vividness subscale of the VCI tests depending upon which hypnotic susceptibility group they were in: the medium and high subjects scored higher on both vividness tests than the low subjects. However, this difference was not statistically significant. The subjects in the Hypnosis-First group also scored differently on their vividness subscales of the VCI depending upon which hypnotic susceptibility group they were in: the high subjects scored significantly higher than the medium subjects who scored significantly higher than the low subjects.

In Coe et al.'s second experiment for both the Hypnosis-only groups and the Waking-Only groups the high subjects scored significantly higher on their vividness scores than the medium subjects who scored significantly higher than the low subjects.

CORRECTION REGARDING OMISSION OF HEALTH STATUS QUESTIONNAIRE ORIGIN

The Health status Questionnaire is a modified version of the Health Status Questionnaire designed and used by C.R. Meager, $198 \emptyset$ (Recall and recognition under varying conditions of hypnotically suggested amnesia. Unpublished Master's Thesis. Portland State University, Portland, Oregon). 


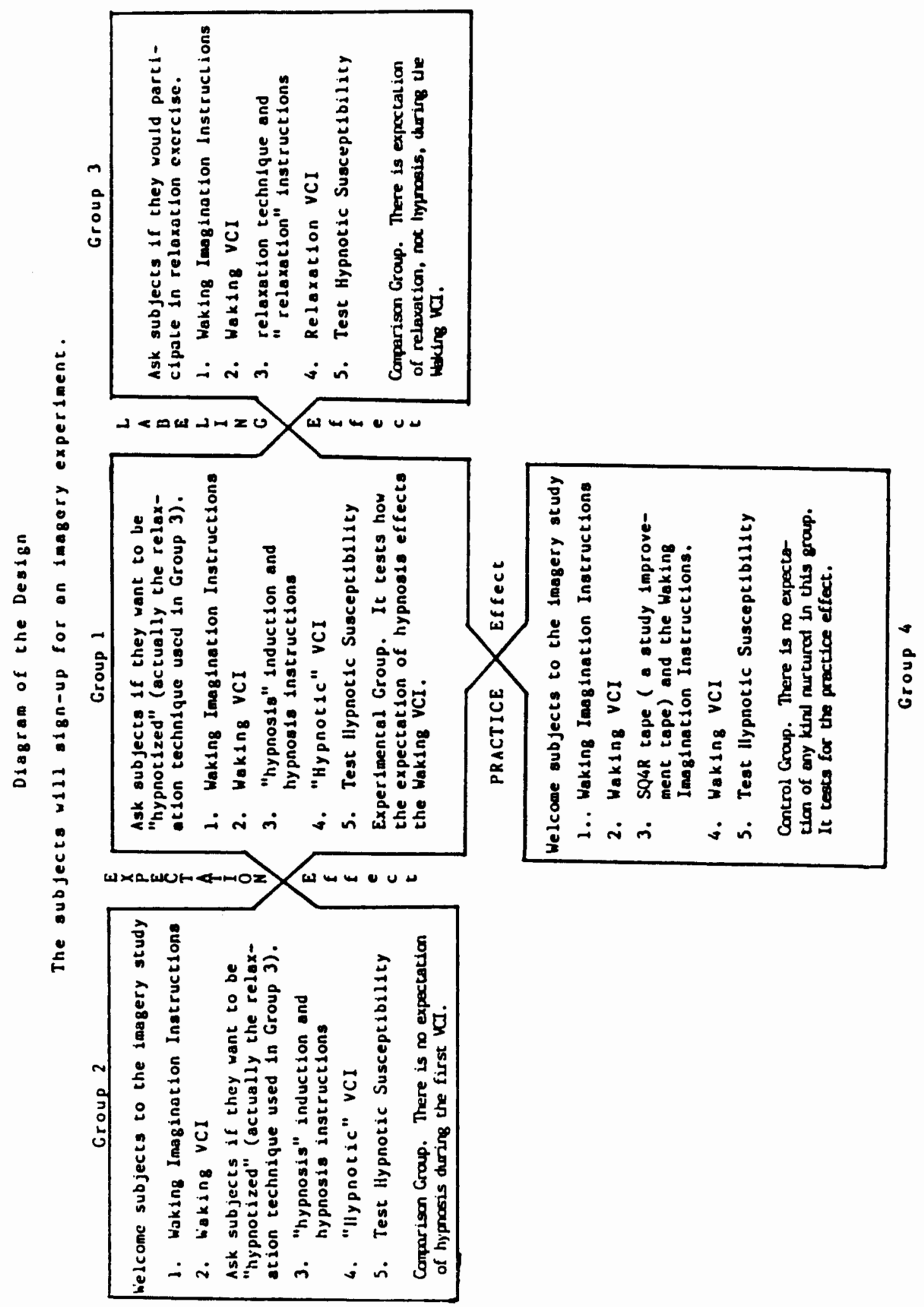




\section{APPENDIX B}

\section{HEALTH STATUS QUESTIONNAIRE}

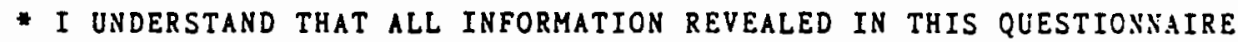
WILL BE KEPT STRICTLY CONFIDENTIAL.

Name:

Age: Sex :

Address:

Street City 2 ip

Phone: Best time to call:

Class/ Instructor:

1. Have you ever had any of the following medical problems?

Epilepsy

Rheumatic

fever

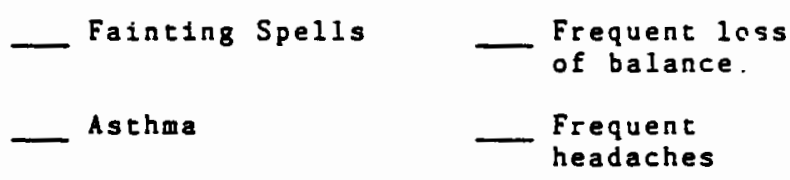

2. Have you ever suffered a severe head injury resulting in loss of consciousness, skull fracture, concussion, or brain danage?

3. Have you ever had a disturbed reaction to hallucinogenic drugs?

4. Are you currently taking any medtcations?

If yes, please specify.

5. Have you ever been treated for emotional problems? If yes, please specify.

6: Have you ever had an unpleasant experience with chemical anesthesia? 


\section{APPENDIX C}

\section{CONSENT FORMS}

Informed Consent Sheet for Group 1

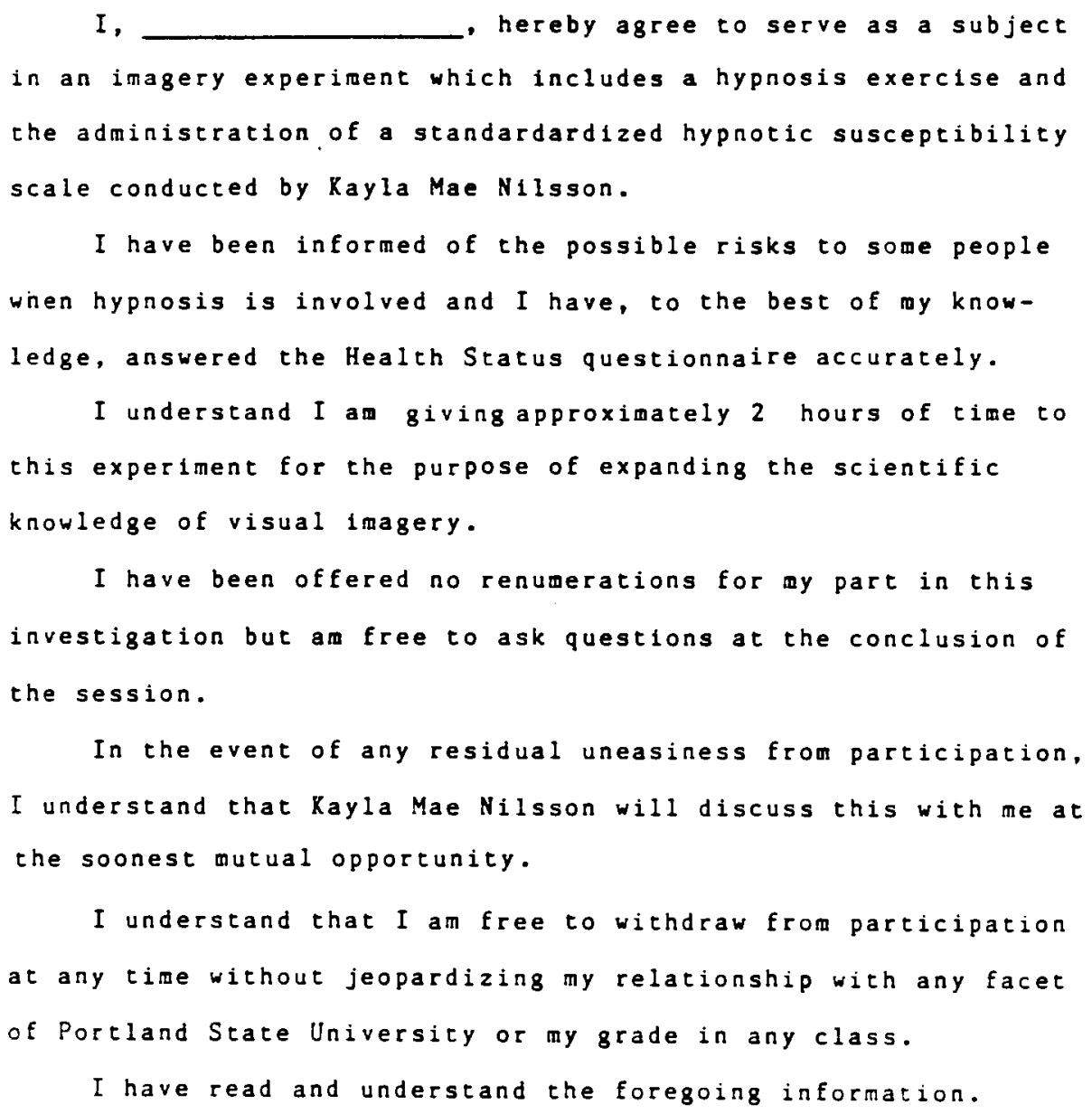


Informed Consent Sheet for Part 1 of Groups 2 and 4

I, hereby agree to serve as a subject in

the experiment involving an imagery task conducted by Kayla Mae Nilsson.

I have been informed of the possible risks, and I have to the best of my knowledge, answered the Health Status questionnaire accurate1y.

I understand I am giving approximately 2 hours of time to this experiment for the purpose of expanding the scientific knowledge of visual imagery.

I have been offered no renumerations for my part in this investigation but am free to ask questions at the conclusion of the session.

In the event of any residual uneasiness from participation, I understand that Rayla Mae Nilsson will discuss this with me at the soonest mutual oppcrtunity.

I understand that I am free to withdraw from participation at any time without jeopardizing my relationship with any facet of Portland State University or my grade in any class.

I have read and understand the foregoing information.

Experimenter

Subject

Date

If you experience problems that are the result of your participation in this study, please contact me, Kayla Nilsson, at the Psychology department 229-3923. 
Informed Consent Sheet for Part 1 of Group 3

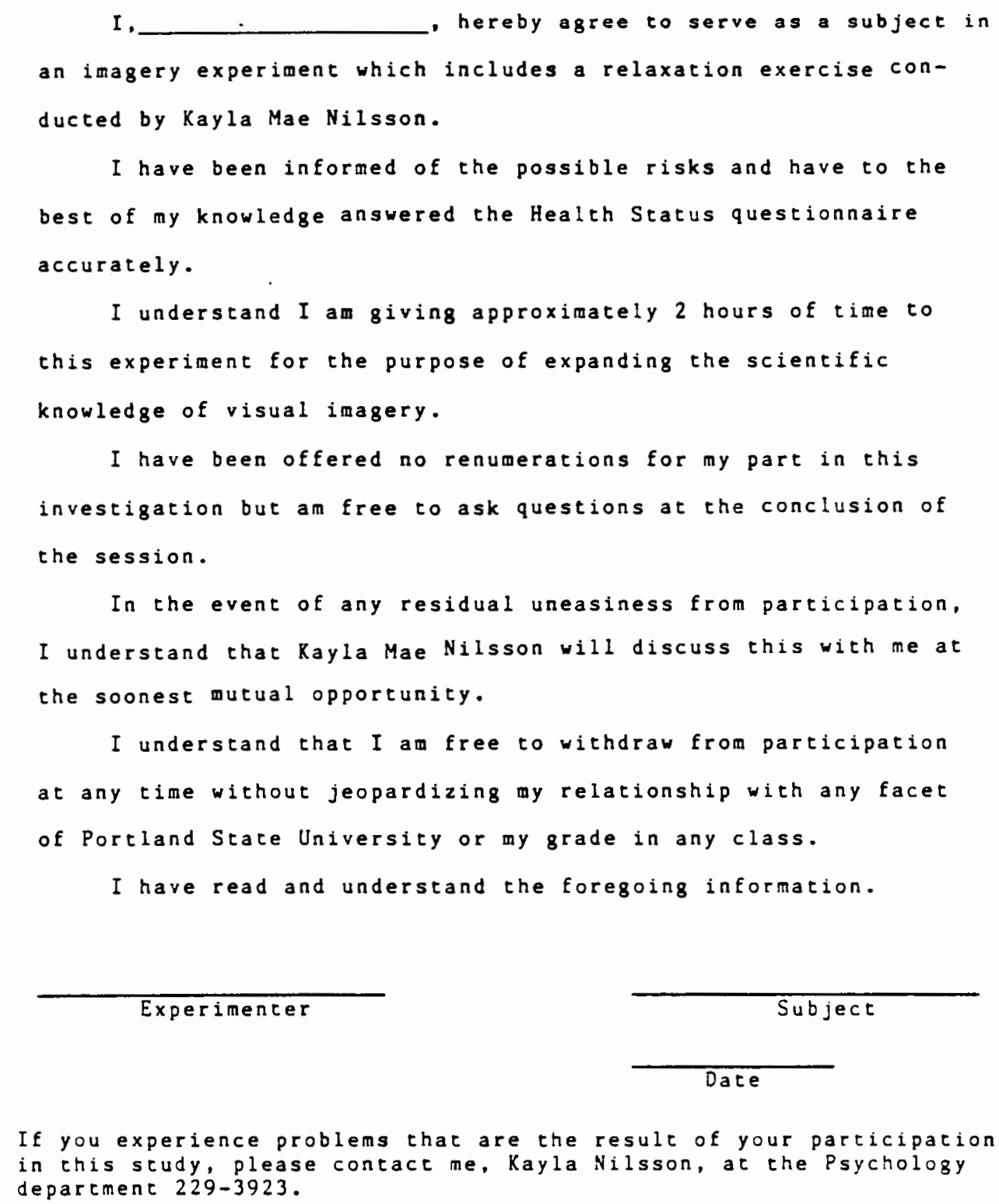

In the event of any residual uneasiness from participation, I understand that Kayla Mae Nilsson will discuss this with me at the soonest mutual opportunity.

I understand that I am free to withdraw from participation at any time without jeopardizing my relationship with any facet of Portland State Universicy or my grade in any class.

I have read and understand the foregoing information.

Experimenter

Subject

\section{Date}

If you experience problems that are the result of your participacion in this study, please contact me, Kayla Nilsson, at the Psychology department 229-3923. 
Informed Consent Sheet for Part 2 of Group 2

I. hereby agree to serve as a subject in the second part of the imagery experiment which involves a hypnosis exercise and the administration of a standardized hypnotic susceptibility scale conducted by Kayla Mae Nilsson.

I have been informed of the possible risks to some people when hypnosis is used and understand $I$ am giving time for the purpose of expanding the scientific knowledge. I also understand I am free to ask questions at the conclusion of the session.

In the event of any uneasiness from participation, I understand that Kayla Mae Nilsson will discuss this with me at the soonest qutual opportunity.

I understand that $I$ am free to withdraw from participation at any time without jeopardizing my relationship with any facet of Portland State University or my grade in any class.

I have read and understand the foregoing information.

Experimenter 
Informed Consent Sheet for Part 3 of Groups 3 and 4

$$
\text { I. }
$$
- hereby agree to serve as a subject in the second part of the imagery experiment which involves the administration of a standardized hypnotic susceptibility scale conducted by Rayla Mae Nilsson.

I have been informed of the possible risks to some people when hypnosis is used and understand $I$ am giving time for the purpose of expanding the scientific knowledge. I also understand I am free to ask quesitong at the conclusion of the session In the event of an uneasiness from participation, I

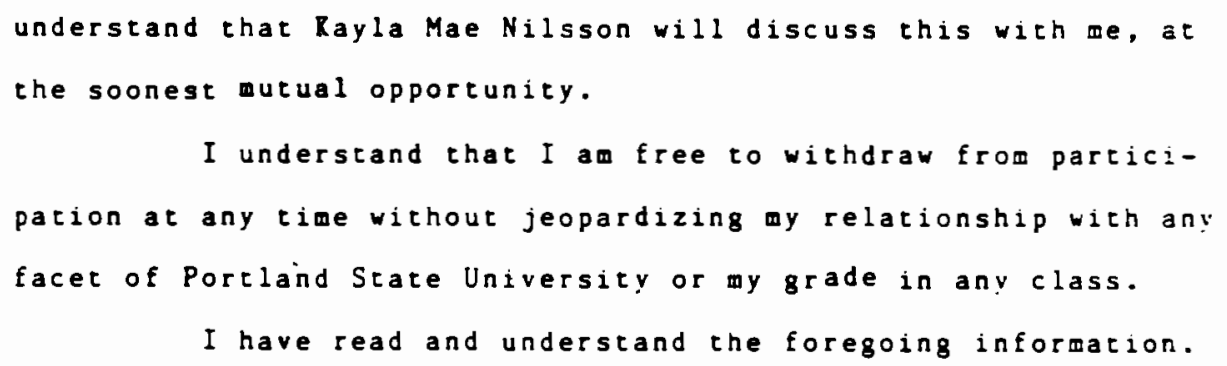




\section{APPENDIX D}

MEASURES USED

The Vividness Section of the

Vividness and Control of Imagery Scale (VCI)

\section{Vividness Instructions}

"In a moment I am going to ask you to imagine some things and then ask you to show me how vivid they seemed to you by holding up the appropriate number of fingers. You will let me know how vivid your images are by showing me a number between one and five. If you hold up one finger, it means that your image was not vivid at all, it was really no different from just thinking about it. If you hold up five fingers, it means that your image was very vivid, just as vivid as if you had really seen it with your normal vision. Numbers 2, 3, and 4 fall in between with each higher number meaning that your image was increasingly vivid. Remember, when I ask you how vivid your image is, show me a number between one and five with your fingers. One meaning not at all vivid, and five meaning very vivid."

(Scene 1 and Questions 1-4 were used as a rehearsal and were not included in the data analysis.)

"Now imagine a relative or friend whom you frequently see and carefully consider the picture that comes before your mind's eye." (Allow 30 seconds). 
1. How vivid is the contour of the face, head, shoulders, and body?"

2. "How vivid is the characteristic pose of the head and attitude of the body?n

3. "How vivid is the carriage of the body and length of step when walking?"

4. "How vivid are the colors of the clothes?"

"Now imagine the sun rising into a stormy sky with a rainbow to one side. Carefully consider the picture that comes before your mind's eye." (Allow 30 seconds)

5. "How vivid is the sun as it rises above the horizon?"

6. "How vivid are the clouds?"

7. "How vivid are the flashes of lightning?"

8. "How vivid is the rainbow?"

"Now imagine you are standing in front of a window display of a store you often go to. Carefully consider the picture that comes before your mind's eye." (Allow 30 seconds)

9. "How vivid is the overall appearance of the store from where you are standing?"

10. "How vivid are the shapes and details of the individual items in the window?"

11. "How vivid are the colors of the items?n 
"Now imagine a country scene which involves trees, mountains, and a lake. Carefully consider the plcture that comes before your mind's eye." (Allow 30 seconds)

12. "How vivid are the contours of the landscape?"

13. "How vivid are the shapes of the trees?"

14. "How vivid are the colors of the trees?"

15. "How vivid is the color and shape of the lake?" 
Instructions for the VCI

\section{Waking Imagination Instructions}

Now just sit back comfortably in your chair and let yourself relax. Just close your eyes and think of your body letting go, relaxing completely... Good, eyes closed and relaxing. You will be able to imagine quite vividly if you just ignore everything except what I ask you to imagine. Just let yourself go and become completely absorbed in each image, like it is actually real. Relax and turn all your attention to what you are imagining, and you will find that you can imagine quite vividly.

\section{Hypnotic Instructions}

In a moment I am again going to ask you to imagine some things. Like before, I want you to show me a number between one and five with your fingers when I ask you how vivid your image is. Remember, one means not at all vivid and five means very vivid. You will remain deeply hypnotized. Now, relax completely--deep, deep relaxation. You will be able to imagine quite vividly and show me how vivid your images are. Ignore everything except what $I$ ask you to imagine. You will be able to just let yourself go and become completely absorbed in each image, like it is actually real. Remain deeply hypnotized, relax and turn all your attention to what you are imagining but hear clearly what I am saying, and you will find that you can imagine quite vividly. 


\section{"Relaxation" Instructions}

In a moment I am again going to ask you to imagine some things. Like before, I want you to show me a number between one and five with your fingers when I ask you how vivid your image is. Remember, one means not at all vivid and five means very vivid. You will remain deeply relaxed. Now, relax completely--deep, deep relaxation. You will be able to imagine quite vividly and show me how vivid your images are. Ignore everything except what $I$ ask you to imagine. Your will be able to just let yourself go and become completely absorbed in each image, like it is actually real. Remain deeply relaxed, relax and turn all your attention to what you are imagining, but you can hear clearly what I am saying, and you will find that you can imagine quite vividly. 


\section{The Relaxation Technique}

Begin by getting as comfortable as you can. Settle back comfortably. Just try to let go of all the tension in your body. Now take in a deep breath. Breath right in and hold it (five-second pause). And now exhale. Just let the air out automatically and feel a calmer feeling beginning to develop. Now just carry on breathing normally and just concentrate on feeling heavy all over in a pleasant way. Study your own body heaviness. This should give you a calm and reassuring feeling all over (ten-second pause). Now let us work on tension and relaxation contrasts. Try to tense every muscle in your body. Every muscle: your jaws, tighten your eyes, your shoulder muscles, your arms, chest, back, stomach, legs, every part tensing and tensing. Feel the tension all over your body--tighter and tighter--tensing everywhere, and now let it go, just stop tensing and relax. Try to feel this wave of calm that comes over you as you stop tensing like that. A definite wave of calm (ten-second pause).

\section{Insert for Relaxation Group (Group 3)}

Your ability to be relaxed depends partly on your willingness to cooperate and partly on your ability to concentrate upon my words. You have already shown yourself to be cooperative by continuing in the experiment, and with your further cooperation I can help you to become relaxed. 
Insert for "Hypnosis" Groups (Groups 1 \& 2)

Your ability to be hypnotized depends partly on your willingness to cooperate and partly on your ability to concentrate upon my words. You have already shown yourself to be cooperative by continuing in the experiment, and with your further cooperation I can help you to become hypnotized.

Now I want you to notice the contrast between the slight tensions that are there when your eyes are open and the disappearance of these surface tensions as you close your eyes. So while relaxing the rest of your body just open your eyes and feel the surface tension which will disappear when you close your eyes. Now close your eyes and feel the greater degree of relaxation with your eyes closed (ten-second pause) all right, let us get back to breathing. Keep your eyes closed and take in a deep, deep breath and hold it. Now relax the rest of your body as well as you can and notice the tension from holding your breath. Study the tension. Now let out your breath and feel the deepening relaxation--just go with it relaxing now. Breathe normally and just feel the relaxation flowing into your forehead and scalp. Think of each part as I call it out--just relaxing-just letting go, easing up, eyes and nose, facial muscles. You might feel a tingling sensation as the relaxation flows 
in. You might have a warm sensation. Whatever you feel I want you to notice it and enjoy it to the fullest as the relaxation now spreads into the face, into the lips, jaws, tongue, and mouth so that your lips are slightly parted as the jaw muscles relax further and further. The throat and neck relaxing (five-second pause), shoulders and upper back relaxing, further and further, feel the relaxation flowing into your arms and to the very tips of your fingers (fivesecond pause). Feel the relaxation in your chest as you breathe regularly and easily. The relaxation spreads even under your armpits and down your sides, right into the stomach area. The relaxation becomes more and more obvious as you do nothing but just give way to the pleasant, serene emotions which fill you as you let go more and more. Feel the relaxation--stomach and lower back all the way through in a warm penetrating, wavy calm and down your hips, buttocks, and thighs to the very, very tips of your toes. The waves of relaxation just travel down your calves to your ankles and toes. Feel relaxed from head to toe.

Now to increase the feelings of relaxation at this point what I want you to do is just keep on relaxing and each time you exhale, each time you breathe out for the next minute, I want you to think the word relax to yourself. Just think the word relax as you breathe out. Now just do that for the next minute (one-minute pause). Okay, just 
feel that deeper relaxation and carry on relaxing. You should feel a deeper, deeper feeling of relaxation. To even further increase the benefits, I want you to feel the emotional calm, those tranquil and serene feelings which tend to cover you all over inside and out, a feeling of safe security, a calm indifference. Relaxation will let you arrive at feeling a quiet inner confidence--a good feeling about yourself (five-second pause). Now once more feel the heavy sensations that accompany relaxation as your muscles switch off so that you feel in good contact with your environment, together, the heavy good feeling of feeling yourself calm and secure and very, very, tranquil and serene.

Now we can deepen the relaxation still further by just using some very special stimulus words. Let's use the words calm and serene. What I would like you to do is to think these words to yourself twenty times or so. Don't bother to count. Approximately, twenty or thirty times just say to yourself calm and serene and then feel the deepening--ever, ever deepening--waves of relaxation as you feel so much more calm and serene. Now you just do that; take your time, think of the words and feel the sensations over and over (pause of about one minute). Good. 
(Administration of the VCI Instructions and the VCI) Now I am going to count backward from 10 to 1 . At the count of 5 I would like you to open your eyes, and then by the time I reach 1, just kind of stretch and yawn. Okay, now counting backward: $10,9,8,7,6,5$, open your eyes, 4 , 3,2 , and 1 . Now just stretch and kind of yawn. 


\section{S04R}

one of the fastest ways to spend less time reading assignments is to figure out what important questions you need to answer while reading. You can then read quickly by gearing your reading to finding answers to your questions. Reading quickly should not be difficult if you consider that a large percentage (perhaps as much as 80 percent) of the words you read are redundant. Most words simply link ideas and concepts. The ideas and concepts are the answers to the questions you wish to answer. Thus, your reading in most instances should be geared to looking for ideas and concepts which answer important questions. Dozens of studies with college students show that the following steps will increase your reading speed, comprehension, and memory: Survey-Question--Read--Recite--Write--Review equals the SQ4R method.

This approach to reading is considered by many experts on study skills and reading improvement to be the most efficient and effective means for getting the most out of reading material in the least time. The primary concern of students using this method will be to ask and answer intelligent questions as they read.

What you should do is...

Survey and Question

The goal of surveying is to determine what important questions are answered in the textbook chapter. First, go 
to the beginning and end of the chapter to see whether or not there are chapter objectives, a list of questions, or a chapter summary. If so, read them right away! This is where you will find the important points the authors wish to stress and the questions students should be able to answer after completing the chapter.

If you can answer the questions and already know what is in the summary of chapter objectives, you probably won't have to read the chapter. But don't decide yet. If there is a set of questions, a list of objectives, or a chapter summary, you're ahead of the game; if not, you soon will be.

How do you survey? The process of surveving involves qui ickly skimming the chapter to determine what importaat questions it answers. Look for titles, subtitles, illustrations, pictures, charts, lead sentences in paragraphs, and questions that will give you a basic idea of what the chapter is about.

While surveying it is easy to turn titles, subtitles, and lead sentences into questions. By generating questions as you survey, you keep yourself alert to the important points in the chapter. Reading becomes an active, goaloriented process. As you survey, you should formulate questions that, when answered, will give you a good summary 
of the chapter. The result of your survey will be a list of questions.

To prove your brilliance, you may wish to attempt to answer the questions you have generated in your survey before reading. This attempt serves to tell you how much you already know before spending an exorbitant amount of time reading. Many students are amazed at their ability to answer a large percentage of the questions they have formulated in their survey.

Another helpful technique is to quickly summarize what you already know about the chapter. By talking to yourself about the chapter, you help yourself to focus on the important questions you should be able to answer after having read it.

When reading, read as quickly as you can. Read to find the answers to the questions you have generated while surveying the chapter and to find new questions and answers that you haven't predicted while surveying.

Remember: In many instances, your questions and answers will be summarized in titles, subtitles, or lead sentences. Occasionally, you may have to read beyond these headings for more important details. But not with the regularity that caused you to waste a lot of time in the past when you were looking for unimportant details.

When reading to answer questions, you learn to predict important questions before spending a lot of time reading. 
You learn to read selectively. You read to find answers to questions. When you come to the answer to a question that you had not predicted, you simply slow down, formulate the question, and make sure you know the answer. When you come to material you already know, keep on going to find out what you don't know.

Now that you have (1) read to answer the questions from your survey and (2) have developed new questions and answers that you had not predicted, it is important to go one step further.

Recite and write the answers to the questions that you developed wile surveying and reading. Equally, important, reccite and write a short summary of what you hay he jus These procedures are excellent means of proving to yourself that you have asked and answered the important questions from each chapter.

Practice talking to yourself about the answers to your questions (even if people think you're a little crazy). often students rush on to a new chapter before thoroughly proving to themselves that they are familiar with the contents of the chapter they just read. They say to themselves, "I read it. I know what it's about." DON'T MAKE THAT MISTAKEI Prove to yourself by answering questions and writing chapter summaries that you really do comprehend the chapter. 
If you have followed the steps so far, you are in excellent shape to review the chapter at any time. You will have a set of questions and answers representing the contents of the chapter. When preparing for your exam, quiz yourself on these questions until you feel comfortable that you could give accurate answers to them if they were to appear on your exam.

You may also summarize to yourself, orally or in writing, the contents of the chapter and compare your summary with the one you wrote after having read the chapter.

Taken together, these activities will really give you the feeling that you've mastered the material. When you know you can answer the questions correctly and make accurate summaries, you will be more confident that you have mastered the chapter. You will spend less time attempting to reread chapters and otherwise involving yourself in a variety of superstitious and time-consuming study activities which seldom help you to ask and answer important questions.

The Result: You have

1. Surveyed the chapter.

2. Generated questions.

3. Read selectively to answer the questions in greater detail.

4. Found questions and answers that you had not predicted.

5. Recited and written answers to questions and chapter summaries.

6. Reviewed the chapter by practicing answering questions and summarizing the chapter. 
You should now have a good understanding of the chapter.

With SQ4R, you spend less time memorizing facts that you will soon forget. You don't waste time reading and looking for things you already know. Your preparation for tests is a continual process. By the time you take the test, you will find that you have answered most of the questions. You focus on grasping the key concepts. Details are then much easier to remember. You don't waste time looking for details that are unimportant to you or your instructor. You learn to take an expert's point of view and to think things out for yourself. You learn to sit down and generate answers that you didn't think you knew. You then search for additional information, which makes polished answers out of incomplete ones. You learn to organize and structure your studying. You state your goals as questions, seek answers, achieve your goals, and move on. 


\section{APPENDIX E}

\section{PROCEDURES USED}

\section{Subject Recruitment Procedure}

Hello, my name is Kayla Nilsson, and $I$ am doing research here at Portland state in the area of visual imagination. Specifically, I am interested in how vivid people's visual images are, and if this vividness can be changed or improved. I will need 80 people who are 18 years of age or older to participate in my experiment which consists of one two hour session. Although participation is not required of any class member, if you do volunteer, it will give you insight into how an experiment is designed and carried out, and how theoretical ideas are tested in psychology. [The following passage was added for classes in which the instructor gave extra-credit for participation in the experiment: has agreed to allow extracredit in this class for your participation, but participation is not required of any class member.]

All the participants will be informed of the rationale of the experiment following the completion of their session, and the results will be made available to them when all the data has been collected and analyzed. All information about the volunteers will remain strictly confidential, including the information on the questionnaire I am about to pass out. 
It asks questions about your physical and emotional health and is required as a precautionary measure by the University.

If you are interested in participation, please raise your hand, and I will pass you a Health status Questionnaire. On this sheet are spaces for your phone number and the best time to call. Please, make sure these are filled out. I will call you for an appointment in the latter part of next week. Four people can take part in the study at one time, so some people may find themselves scheduled for the same session.

Because of the design of the study, not everyone who volunteers will be called for an appointment. Are there any questions?

Thank you for your time. When you are finished filling out the questionnaires, please, fold them in half, and pass them to the front. 


\section{Subject Debriefing Procedure}

Thank you for participating in my research project. The purpose of this experiment was to determine if people's expectations of hypnosis affected their vividness of imagery scores. There are four different groups in this experiment. You are in Group

In Groups 1 and 2 the people participated in a hypnosis exercise before the second imagery test. Group 1 was told about the hypnosis at the beginning of the session, and Group 2 was told about the hypnosis after they took the first, waking imagery test. Therefore, people's expectations in Group 1 would affect their first imagery scores, but people's expectations in Group 2 would not affect the first Imagery task. So, I can compare the imagery scores of these two groups to see if people's expectations do affect their visual imagery scores.

In Group 3 the participants were told they would participate in a relaxation technique and take a visual imagery test after they took the first, waking imagery test at the beginning of the session (like in Group 1). Therefore, their expectations of being relaxed would affect their first imagery scores. So, the imagery scores in Group 3 can be compared to Group 1 to see if people's expectations of relaxation are as strong as their expectations of hypnosis. 
Group 4 was the control group; they were not hypnotized or relaxed. They took two waking imagery tests, and, to keep this group comparable to the other three groups, they were given a tape on improving study skills. The scores from this were used to see if the results in the other groups were changed by the practice effect of taking the same imagery test twice.

At the end of the session all of the people in the four groups took a standardized hypnotic ability test.

In short, Group 1 and Group 3 expected either hypnosis or relaxation during the first imagery test, and Group 2 and Group 4 did not. The imagery scores for these groups will be compared.

In order to make sure that the difference in scores between the two imagery tests were due solely to expectations, not the effects of hypnosis, Groups 1 and 2 participated in a relaxation technique labeled "hypnosis," the same as the one used in Group 3. [The following passage was added to the debriefing procedures of Groups 1 and 2: However, since there is a family resemblance between hypnotic inductions and relaxation instructions, some people become hypnotized when listening to instructions which were originally designed for relaxation. I asked for your consent to be hypnotized even though you were listening to relaxation instructions. For some people there is not much difference between the two states.] 
The standardized hypnotic ability test was an actual hypnosis induction, and your scores are a good indication of your hypnotic ability. So, if you would like to know your hypnotic ability score, please tell me now, and I will give you a post card. Please, put your name and address on the front and give it back to me. I will mail them after everyone has been tested.

Because of the nature of my experiment (that is the need to set up different expectations at different times in different groups), I must ask you PLEASE DO NOT TALK ABOUT THIS EXPERIMENT WITH ANYONE OTHER THAN MYSELF, IN PRIVATE, UNTIL THE EXPERIMENT HAS BEEN COMPLETED. This will be, probably, by the beginning of fall term. Please, remember that even the mere MENTION of the word HYPNOSIS in connection with this experiment would ruin the study. So, please help me by not saying anything about it. If you are asked, please, simply say that you were asked not to discuss it until everyone has participated.

Your results will be combined with the people in the other groups, and the final analysis will be mailed to you if you are interested. All individual results will be kept strictly confidential.

If you have any residual uneasiness or have concerns about any of the procedures used, you may contact me through the Psychology Department. 
If you participated in this experiment for extra credit, I will now give you a silp to hand to your instructor as proof of participation.

Thank you! 


\section{Experimental Procedure for Group 1}

[Greet participants]. Hello, as you may remember my name is Kayla Nilsson, and $I$ am interested in visual imagery. I would like you to participate in two visual imagery tests. I am also interested in how visual imagery is affected during hypnosis; therefore, I would like you to participate in a hypnosis exercise, but this will happen after you've taken the first imagery test. I'd also like you to take a standardized hypnotic ability test at the end of the session. After the entire experiment is over, I will be able to mail you your hypnotic ability scores.

For almost everyone, hypnosis is a pleasant and interesting experience. However, some people may feel dizzy, may experience a loss of balance, or may feel confused for a short time. The Health Status Questionnaire you filled out shows that it is very unlikely you will have any bad effects. When people experience bad effects from hypnosis, it is almost always because they were exploring their past or some other emotionally-weighted material. We will not be doing this. I am interested in how hypnosis affects your visual images of standardized scenes, and how much hypnotic ability you have. There will be nothing in the procedure to make you look silly or feel foolish. With that in mind, please read over the consent form [hand out consent forms]. If you wish to participate, please sign it 
and hand it to me. If you do not wish to participate, thank you for your time. You will receive credit for the experiment.

Now you will take the first, waking imagery test. There will be four scenes to imagine, and there will be a pause after the description of each scene. After this pause, I will ask some questions about how well you imagined the scene. Since your eyes will be closed during this test, and there are other people in the room whom you do not want to disturb, you will tell me how vivid your images are by showing me a number from 1 to 5 with your fingers. One means the image is not vivid at all, and five means the image is very vivid. The bigger the number, the more vivid the image. When you show your fingers, please keep them up until you hear the next question.

\section{Part 2}

Now you will be hypnotized and take the second imagery test. There will be no space between the hypnotic exercise and the imagery test. Remember, I am interested in how hypnosis affects the vividness of your visual images of standardized scenes, and you will not be asked to do anything foolish, silly, or personal. Please, also remember there are people around you trying to concentrate. Please, try not to fall asleep. If you fall asleep, I will nudge you, then please continue answering the questions. I will 
answer any questions now before I turn on the tape-recorder for the second imagery task during hypnosis.

\section{Part 3}

As I said at the beginning, I am also interested in how hypnotic ability affects the vividness of visual imagery. I would like you to take a hypnotic ability test now. If you wish, I will send your score later.

I will now pass out the booklet for a test of hypnotic susceptibility. Please, do not open your booklet until I tell you to do so. Please, fill out the information requested. All information will be kept confidential.

The hypnotic ability test has also been pre-recorded to keep it constant, so there will be no further discussion or questions after I begin the tape. [The introduction from the HGSHS-A will then be given, any questions answered, and the tape will be turned on.] 


\section{Experimental Procedure for Group 2}

[Greet Participants]. Hello, as you may remember my name is Kayla Nilsson, and I am interested in visual imagery. I would like you to participate in two visual imagery tests [hand out consent forms]. I am handing out some consent forms. Please read them and sign them if you agree. Then, hand them to me. Now you will take the first imagery test.

There will be four scenes to imagine, and there will be a pause after the description of each scene. After this pause, I will ask some questions about how well you imagined the scene. Since you eyes will be closed during this test, and there are other people in the room whom you do not want to disturb, you will tell my how vivid your images are by showing me a number from 1 to 5 with your fingers. One means the image is not vivid at all, and five means the image is very vivid. The bigger the number, the more vivid the image. When you show your fingers, please keep them up until you hear the next question.

All the procedures in the experiment are pre-recorded to keep the procedures constant, so I will answer any questions now.

Sometimes people tend to talk or be amused when doing something new and unfamiliar, so please, try not to talk or giggle during the recording; remember, others are trying to 
concentrate. I will now turn on the tape-recorder for the first imagery test.

\section{Part 2}

I am also interested in how visual imagery is affected during hypnosis; therefore, I would like you to participate in a hypnosis exercise and then take the second imagery test. I'd also like you to take a standardized hypnotic ability test at the end of the session. After the entire experiment is over, I will be able to mail you your hypnotic ability scores.

For almost everyone, hypnosis is a pleasant and interesting experience. However, some people may feel dizzy, may experience a loss of balance, or may feel confused for a short time. The Health Status Questionnaire you filled out shows that it is very unlikely you will have any bad effects. When people experience bad effects from hypnosis, it is almost always because they were exploring their past or some other emotionally-weighted material. We will not be doing this. I am interested in how hypnosis affects your visual images of standardized scenes, and how much hypnotic ability you have. There will be nothing in the procedure to make you look silly or feel foolish. With that in mind, please read over the consent form [hand out consent forms]. If you wish to participate, please sign it and hand it to me. If you do not wish to participate, thank 
you for your time. You will receive credit for the experiment.

Now you will be hypnotized and take the second imagery test. There will be no space between the hypnotic exercise and the imagery test. Remember, I am interested in how hypnosis affects the vividness of your visual images of standardized scenes, and you will not be asked to do anything foolish, silly, or personal. Please, also remember there are people around you trying to concentrate. Please, try not to fall asleep. If you fall asleep, I will nudge you, then please continue answering the questions. I will answer any questions now before I turn on the tape-recorder for the second imagery task during hypnosis.

\section{Part 3}

As I said at the beginning, I am also interested in how hypnotic ability affects the vividness of visual imagery. I would like you to take a hypnotic ability test now. If you wish, I will send your score later.

I will now pass out the booklet for a test of hypnotic susceptibility. Please, do not open your booklet until I tell you to do so. Please, fill out the information requested. All information will be kept confidential.

The hypnotic ability test has also been pre-recorded to keep it constant, so there will be no further discussion or questions after I begin the tape. [The introduction from 
the HGSHS-A will then be given, any questions answered, and the tape will be turned on.] 


\section{Experimental Procedure for Group 3}

[Greet Participants]. Hello, as you may remember my name is Kayla Nilsson, and $I$ am interested in visual imagery. I would like you to participate in two visual imagery tests. I am also interested in how visual imagery is affected during relaxation; therefore, I would like you to participate in a relaxation exercise, but this will happen after you have taken the first imagery test.

For almost everyone, relaxation is a pleasant and interesting experience. However, some people may feel dizzy, may experience a loss of balance, or may feel confused for a short time. The Health Status Questionnaire you filled out shows that it is very unlikely you will have any bad effects. When people experience bad effects from relaxation, it is almost always because they were exploring their past or some other emotionally-weighted material. We will not be doing this. I am interested in how relaxation affects your visual images of standardized scenes. There will be nothing personal about the procedure. With that in mind, please read over the consent form [hand out consent forms]. If you wish to participate, please sign it and hand it to me. If you do not wish to participate, thank you for your time. You will receive credit for the experiment.

Now you will take the first, waking imagery test. There will be four scenes to imagine, and there will be a 
pause after the description of each scene. After this pause, I will ask some questions about how well you imagined the scene. Since you eyes will be closed during this test, and there are other people in the room whom you do not want to disturb, you will tell my how vivid your images are by showing me a number from 1 to 5 with your fingers. One means the image is not vivid at all, and five means the image is very vivid. The bigger the number, the more vivid the image. When you show your fingers, please keep them up until you hear the next question.

All the procedures in the experiment are pre-recorded to keep the procedures constant, so I will answer any questions now.

Sometimes people tend to talk or be amused when doing something new and unfamiliar, so please, try not to talk or giggle during the recording. Remember, others are trying to concentrate. I will now turn on the tape-recorder for the first waking imagery test.

\section{Part 2}

Now you will be relaxed and take the second imagery test. There will be no space between the relaxation exercise and the imagery test. Remember, I am interested in how relaxation affects the vividness of your visual images of standardized scenes, and you will not be asked to do anything personal. Please, also remember there are people 
around you trying to concentrate. Please, try not to fall asleep. If you fall asleep, I will nudge you, then please continue answering the questions. I will answer any questions now before I turn on the tape-recorder. I will now turn on the tape-recorder for the second imagery task during relaxation.

\section{Part 3}

I am also interested in how hypnotic ability affects vividness of visual imagery, and I would like you to take a hypnotic ability test now. If you are interested, I will send your hypnotic ability score after the entire experiment is over.

Some people may experience the same negative effects from hypnosis as they might from relaxation. However, the precautions taken for the relaxation exercise also protect from the bad effects of hypnosis (i.e. the Health status Questionnaire and the standardized nature of the ability test). Keeping this in mind, please read over the consent form [hand out consent forms], and if you wish to participate, sign it and hand it to me. If you do not want to participate further, thank you for your participation. You will receive credit for the experiment.

I will now pass out the booklet for a test of hypnotic ability. Please, do not open your booklet until I tell you 
to do so. Please, fill out the information requested. All information will be kept confidential.

The hypnotic ability test has also been pre-recorded to keep it constant, so there will be no further discussion or questions after I begin the tape. [The introduction from the HGSHS-A will then be given, any questions answered, and the tape will be turned on.] 


\section{Experimental Procedure for Group 4}

[Greet Participants]. Hello, as you may remember my name is Kayla Nilsson, and $I$ am interested in visual imagery. I would like you to participate in two visual imagery tests. [Hand out consent forms]. I am handing out some consent forms. Please read them and sign them if you agree. Then, hand them to me. Now you will take the first imagery test.

There will be four scenes to imagine, and there will be a pause after the description of each scene. After this pause, I will ask some questions about how well you imagined the scene. Since you eyes will be closed during this test, and there are other people in the room whom you do not want to disturb, you will tell my how vivid your images are by showing me a number from 1 to 5 with your fingers. One means the image is not vivid at all, and five means the image is very vivid. The bigger the number, the more vivid the image. When you show your fingers, please keep them up until you hear the next question.

All the procedures in the experiment are pre-recorded to keep the sessions constant, so I will answer any questions now.

Sometimes people tend to talk or be amused when doing something new and unfamiliar, so please, try not to talk or giggle during the recording; remember, others are trying to 
concentrate. I will now turn on the tape-recorder for the first imagery test.

\section{Part 2}

Now, I would like you to sit back, close your eyes, and listen to the following tape about improving your study habits. You will then take the second imagery test immediately afterwards with no break. Before I start the tape, are there any questions? I will now turn on the tape.

\section{Part 3}

I am also interested in how hypnotic ability affects vividness of visual imagery, and I would like you to take a hypnotic ability test now. If you are interested, I will send you your hypnotic ability score after the entire experiment is over.

For almost everyone, hypnosis is a pleasant and interesting experience. However, some people may feel dizzy, may experience a loss of balance, or may feel confused for a short time. The Health Status Questionnaire you filled out shows that it is very unlikely you will have any bad effects. When people experience bad effects from hypnosis, it is almost always because they were exploring their past or some other emotionally-weighted material. We will not be doing this. I am interested in how much hypnotic ability you have. There will be nothing in the procedure to make you look silly or feel foolish. With that 
in mind, please read over the consent form [hand out consent forms]. If you wish to participate, please sign it and hand it to me. If you do not wish to participate, thank you for your time. You will receive credit for the experiment.

Now I will pass out the answer booklet for the hypnotic ability test. Please, do not open your booklet until I tell you to do so. Please, fill out the information requested. All the information will be kept strictly confidential.

The hypnotic ability test has also been pre-recorded to keep it constant, so there will be no further discussion or questions after I begin the tape. [The introduction from the HGSHS-A will then be given, any questions answered, and the tape will be turned on.] 
APPENDIX F

SUBJECTS' DATA AND CHARACTERISTICS

GROUP 1

Medium Susceptibility Range $4-8$

\begin{tabular}{llllllll} 
Sub. & $\begin{array}{l}\text { Hyp. } \\
\text { Sus. }\end{array}$ & $\begin{array}{l}\text { 1st } \\
\text { VCI }\end{array}$ & $\begin{array}{l}\text { 2nd } \\
\text { VCI }\end{array}$ & $\begin{array}{l}\text { Hyp. } \\
\text { Exp. }\end{array}$ & $\begin{array}{l}\text { Img.t } \\
\text { Exp. }\end{array}$ & Sex & Age \\
\hline 001 & 5 & 29 & 39 & no & no & F & 44 \\
002 & 5 & 41 & 50 & no & yes & F & 21 \\
003 & 7 & 33 & 46 & no & no & M & 32 \\
004 & 8 & 40 & 48 & no & no & M & 27 \\
005 & 8 & 23 & 24 & no & no & F & 26 \\
006 & 5 & 24 & 40 & no & no & M & 19 \\
007 & 7 & 36 & 45 & yes & no & M & 25
\end{tabular}

High Susceptibility Range 9-12

\begin{tabular}{lrrrllll} 
Sub. & $\begin{array}{l}\text { Hyp. } \\
\text { Sus. }\end{array}$ & $\begin{array}{l}\text { 1st } \\
\text { VCI }\end{array}$ & $\begin{array}{l}\text { 2nd } \\
\text { VCI }\end{array}$ & $\begin{array}{l}\text { Hyp. } \\
\text { Exp. }\end{array}$ & $\begin{array}{l}\text { Img. } \\
\text { Exp. }\end{array}$ & Sex & Age \\
\hline 008 & 10 & 43 & 50 & yes & no & F & 27 \\
009 & 11 & 32 & 44 & yes & no & F & 31 \\
010 & 10 & 45 & 53 & no & no & M & 25 \\
011 & 12 & 31 & 41 & yes & no & M & 33 \\
012 & 9 & 47 & 45 & yes & no & F & 20
\end{tabular}

Low Susceptibility Range $0-3$ [not included in data analysis]

\begin{tabular}{llllllll} 
Sub. & $\begin{array}{l}\text { Hyp. } \\
\text { Sus. }\end{array}$ & $\begin{array}{l}\text { 1st } \\
\text { VCI }\end{array}$ & $\begin{array}{l}\text { 2nd } \\
\text { VCI }\end{array}$ & $\begin{array}{l}\text { Hyp. } \\
\text { Exp. }\end{array}$ & $\begin{array}{l}\text { Img. } \\
\text { Exp. }\end{array}$ & Sex & Age \\
\hline 013 & 2 & 40 & 44 & no & no & F & 41 \\
014 & 3 & 51 & 55 & no & no & F & 23 \\
015 & 3 & 35 & 43 & no & no & F & 20
\end{tabular}

* Subject 011 meditated regularly, and since meditation is closely related to hypnosis, he is categorized as having hypnotic experience.

+ This category is Imagination Experience. It is included because one instructor that term taught his students how to produce vivid images. If they were recruited from his class, they are included in this category. 


\section{GROUP 2}

Medium Susceptibility Range $4-8$

\begin{tabular}{llllllll} 
Sub. & $\begin{array}{l}\text { Hyp. } \\
\text { Sus. }\end{array}$ & $\begin{array}{l}\text { 1st } \\
\text { VCI }\end{array}$ & $\begin{array}{l}\text { 2nd } \\
\text { VCI }\end{array}$ & $\begin{array}{l}\text { Hyp. } \\
\text { Exp. }\end{array}$ & $\begin{array}{l}\text { Img. } \\
\text { Exp. }\end{array}$ & Sex & Age \\
\hline 016 & 6 & 48 & 51 & no & no & M & 47 \\
017 & 5 & 31 & 41 & no & no & F & 24 \\
018 & 8 & 36 & 49 & no & no & F & 32 \\
019 & 5 & 44 & 43 & yes & no & F & 48 \\
020 & 6 & 49 & 53 & no & no & F & 20 \\
021 & 8 & 52 & 53 & no & no & M & 19 \\
022 & 6 & 39 & 45 & no & no & F & 48 \\
023 & 6 & 42 & 45 & no & no & F & 25 \\
024 & 7 & 25 & 28 & no & no & M & 26 \\
025 & 7 & 30 & 36 & yes* & no & M & 22
\end{tabular}

High Susceptibility Range 9-12

\begin{tabular}{llllllll} 
Sub. & $\begin{array}{l}\text { Hyp. } \\
\text { Sus. }\end{array}$ & $\begin{array}{l}\text { 1st } \\
\text { VCI }\end{array}$ & $\begin{array}{l}\text { 2nd } \\
\text { VCI }\end{array}$ & $\begin{array}{l}\text { Hyp. } \\
\text { Exp. }\end{array}$ & $\begin{array}{l}\text { Img. } \\
\text { Exp. }\end{array}$ & Sex & Age \\
\hline 026 & 10 & 48 & 44 & no & no & $M$ & 20 \\
027 & 12 & 37 & 51 & no & no & $M$ & 28 \\
028 & 11 & 35 & 48 & no & yes & $M$ & 29
\end{tabular}

* Subject 025 participated in a relaxation experiment. 
GROUP 3

Medium Susceptibility Range 4-8

\begin{tabular}{llllllll} 
Sub. & $\begin{array}{l}\text { Hyp. } \\
\text { Sus. }\end{array}$ & $\begin{array}{l}\text { 1st } \\
\text { VCI }\end{array}$ & $\begin{array}{l}\text { 2nd } \\
\text { VCI }\end{array}$ & $\begin{array}{l}\text { Hyp. } \\
\text { Exp. }\end{array}$ & $\begin{array}{l}\text { Img. } \\
\text { Exp. }\end{array}$ & Sex & Age \\
\hline 029 & 6 & 32 & 32 & no & no & F & 23 \\
030 & 8 & 38 & 44 & no & no & F & 20 \\
031 & 6 & 47 & 51 & no & no & F & 35 \\
032 & 4 & 35 & 47 & no & no & F & 49 \\
033 & 6 & 39 & 50 & no & no & F & 21 \\
034 & 4 & 37 & 37 & no & no & M & 18 \\
035 & 5 & 11 & 13 & no & no & F & 40 \\
036 & 5 & 34 & 35 & no & no & M & 39 \\
037 & 4 & 40 & 43 & no & no & F & 23 \\
038 & 8 & 25 & 44 & no & no & F & 21 \\
039 & 8 & 39 & 49 & no & no & F & 23
\end{tabular}

High Susceptibility Range 9-12

\begin{tabular}{llllllll} 
Sub. & $\begin{array}{l}\text { Hyp. } \\
\text { Sus. }\end{array}$ & $\begin{array}{l}\text { 1st } \\
\text { VCI }\end{array}$ & $\begin{array}{l}\text { 2nd } \\
\text { VCI }\end{array}$ & $\begin{array}{l}\text { Hyp. } \\
\text { Exp. }\end{array}$ & $\begin{array}{l}\text { Img. } \\
\text { Exp. }\end{array}$ & Sex & Age \\
\hline 040 & 9 & 34 & 42 & no & yes & F & 23 \\
041 & 12 & 35 & 51 & yes* & no & F & 29
\end{tabular}

Low Susceptibility Range $0-3$ [not included in data analysis]

\begin{tabular}{llllllll} 
Sub. & $\begin{array}{l}\text { Hyp. } \\
\text { Sus. }\end{array}$ & $\begin{array}{l}\text { 1st } \\
\text { VCI }\end{array}$ & $\begin{array}{l}\text { 2nd } \\
\text { VCI }\end{array}$ & $\begin{array}{l}\text { Hyp. } \\
\text { Exp. }\end{array}$ & $\begin{array}{l}\text { Img. } \\
\text { Exp. }\end{array}$ & Sex & Age \\
\hline 042 & 3 & 38 & 27 & no & no & F & 30 \\
043 & 3 & 43 & 47 & no & no & F & 25 \\
044 & 3 & 50 & 45 & no & no & M & 29
\end{tabular}

* Subject 040 used a relaxation exercise regularly. 


\section{GROUP 4}

Medium Susceptibility Range 4-8

\begin{tabular}{llllllll} 
Sub. & $\begin{array}{l}\text { Hyp. } \\
\text { Sus. }\end{array}$ & $\begin{array}{l}\text { 1st } \\
\text { VCI }\end{array}$ & $\begin{array}{l}\text { 2nd } \\
\text { VCI }\end{array}$ & $\begin{array}{l}\text { Hyp. } \\
\text { Exp. }\end{array}$ & $\begin{array}{l}\text { Img. } \\
\text { Exp. }\end{array}$ & Sex & Age \\
\hline 045 & 4 & 44 & 49 & yes & no & F & 25 \\
046 & 4 & 39 & 40 & no & no & M & 23 \\
047 & 8 & 37 & 44 & no & no & $M$ & 19 \\
048 & 8 & 39 & 38 & no & no & $M$ & 22 \\
049 & 8 & 29 & 22 & no & no & M & 21 \\
050 & 4 & 41 & 42 & no & no & F & 19 \\
051 & 7 & 36 & 37 & no & no & M & 20 \\
052 & 5 & 31 & 33 & no & no & F & 27 \\
053 & 8 & 35 & 37 & no & no & F & 42 \\
054 & 6 & 41 & 37 & no & no & $M$ & 22
\end{tabular}

High Susceptibility Range 9-12

\begin{tabular}{|c|c|c|c|c|c|c|c|}
\hline Sub. & $\begin{array}{l}\text { Hyp. } \\
\text { Sus. }\end{array}$ & $\begin{array}{l}1 \mathrm{st} \\
\text { VCI }\end{array}$ & $\begin{array}{l}\text { 2nd } \\
\text { VCI }\end{array}$ & $\begin{array}{l}\text { Нyp. } \\
\text { Exp. }\end{array}$ & $\begin{array}{l}\text { Img. } \\
\text { Exp. }\end{array}$ & $\operatorname{sex}$ & Age \\
\hline $\begin{array}{l}055 \\
056\end{array}$ & $\begin{array}{l}9 \\
9\end{array}$ & $\begin{array}{l}42 \\
45\end{array}$ & $\begin{array}{l}52 \\
45\end{array}$ & $\begin{array}{l}\text { yes } \\
\text { yes }\end{array}$ & $\begin{array}{l}\text { no } \\
\text { no }\end{array}$ & $\begin{array}{l}M \\
M\end{array}$ & $\begin{array}{l}18 \\
23\end{array}$ \\
\hline
\end{tabular}

Low Susceptibility Range-0-3 [not included in data analysis]

\begin{tabular}{llllllll} 
Sub. & $\begin{array}{l}\text { Hyp. } \\
\text { Sus. }\end{array}$ & $\begin{array}{l}\text { 1st } \\
\text { VCI }\end{array}$ & $\begin{array}{l}\text { 2nd } \\
\text { VCI }\end{array}$ & $\begin{array}{l}\text { Hyp. } \\
\text { Exp. }\end{array}$ & $\begin{array}{l}\text { Img. } \\
\text { Exp. }\end{array}$ & Sex & Age \\
\hline 057 & 1 & 26 & 36 & no & no & F & 31 \\
058 & 3 & 30 & 31 & no & no & M & 46 \\
059 & 0 & 37 & 34 & no & yes & M & 36 \\
060 & 1 & 40 & 43 & no & no & F & 31 \\
061 & 2 & 36 & 31 & no & no & M & 27
\end{tabular}

* Subject 055 mediated regularly. 


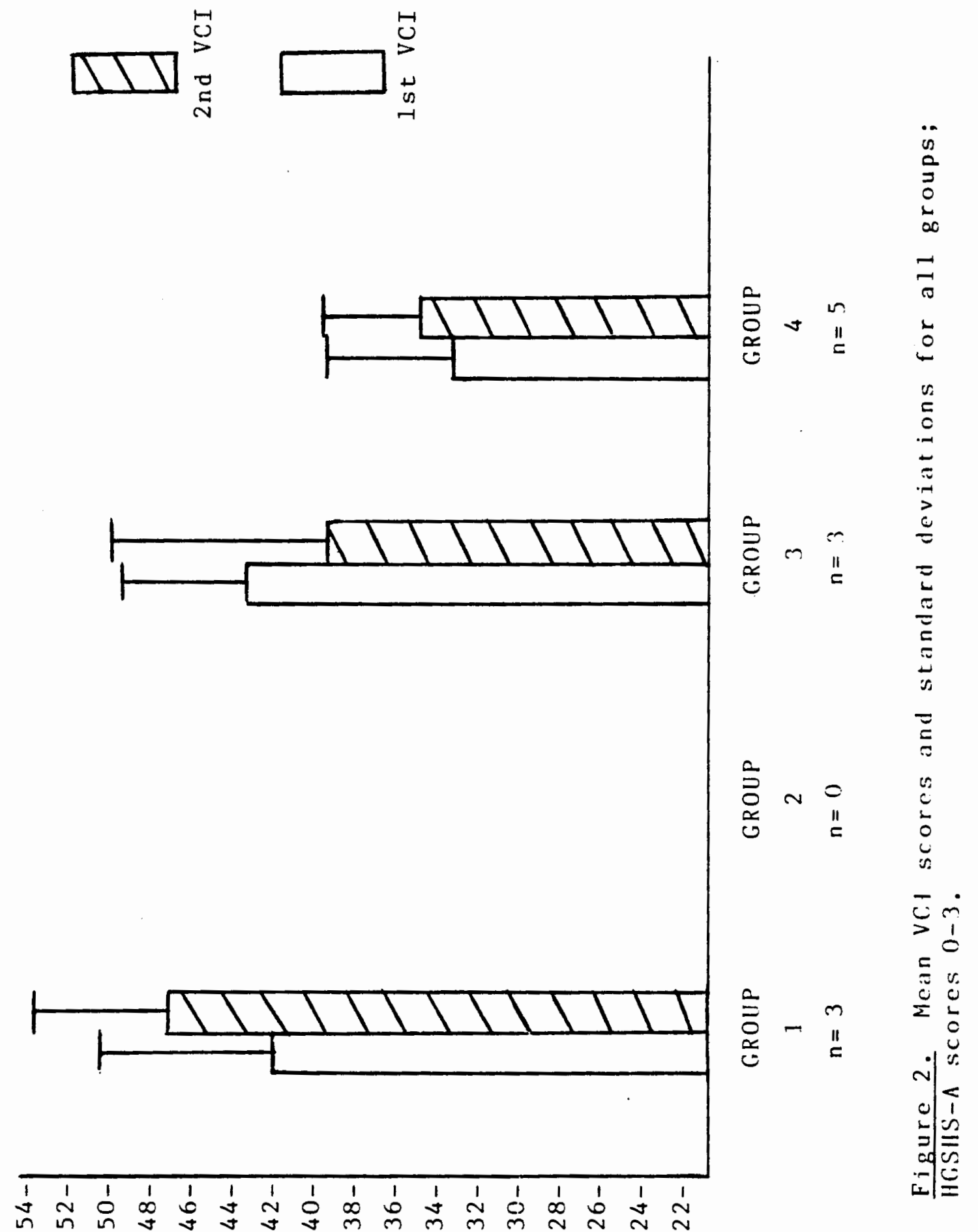

